\title{
Minimal Session Types for the $\pi$-calculus
}

\author{
Alen Arslanagić \\ University of Groningen \\ The Netherlands
}

\author{
Anda-Amelia Palamariuc \\ University of Groningen \\ The Netherlands
}

\author{
Jorge A. Pérez \\ University of Groningen and CWI \\ The Netherlands
}

\begin{abstract}
Session types enable the static verification of message-passing programs. A session type specifies a channel's protocol as sequences of messages. Prior work established a minimality result: every process typable with standard session types can be compiled down to a process typable using minimal session types: session types without the sequencing construct. This result justifies session types in terms of themselves; it holds for a higher-order session $\pi$-calculus, where values are abstractions (functions from names to processes).

This paper establishes a new minimality result but now for the session $\pi$-calculus, the language in which values are names and for which session types have been more widely studied. Remarkably, this new minimality result can be obtained by composing known results. We develop optimizations of our new minimality result, and establish its static and dynamic correctness.
\end{abstract}

\section{CCS CONCEPTS}

- Theory of computation $\rightarrow$ Process calculi; Type structures.

\section{KEYWORDS}

Concurrency, Session Types, Process Calculi, Expressiveness

\section{ACM Reference Format:}

Alen Arslanagić, Anda-Amelia Palamariuc, and Jorge A. Pérez. 2021. Minimal Session Types for the $\pi$-calculus. In 23rd International Symposium on Principles and Practice of Declarative Programming (PPDP 2021), September 6-8, 2021, Tallinn, Estonia. ACM, New York, NY, USA, 15 pages. https://doi.org/10.1145/3479394.3479407

\section{INTRODUCTION}

Session types are a type-based approach to statically ensure correct message-passing programs $[6,7]$. A session type stipulates the sequence and payload of the messages exchanged along a channel In this paper, we investigate a minimal formulation of session types for the $\pi$-calculus, the paradigmatic calculus of concurrency. This elementary formulation is called minimal session types (MSTs).

The gap between standard and minimal session types concerns sequentiality in types. Sequentiality, denoted by the action prefix '; ', is arguably the most distinguishing construct of session types. For instance, in the session type $S=$ ?(Int); ?(Int); !〈Bool〉; end, this construct conveniently specifies a channel protocol that first receives (?) two integers, then sends (!) a Boolean, and finally ends.

Permission to make digital or hard copies of all or part of this work for personal or classroom use is granted without fee provided that copies are not made or distributed for profit or commercial advantage and that copies bear this notice and the full citation on the first page. Copyrights for components of this work owned by others than the author(s) must be honored. Abstracting with credit is permitted. To copy otherwise, or republish, to post on servers or to redistribute to lists, requires prior specific permission and/or a fee. Request permissions from permissions@acm.org.

PPDP 2021, September 6-8, 2021, Tallinn, Estonia

(c) 2021 Copyright held by the owner/author(s). Publication rights licensed to ACM. ACM ISBN 978-1-4503-8689-0/21/09..\$15.00

https://doi.org/10.1145/3479394.3479407
Because sequentiality is so useful for protocol specification and verification, a natural question is whether it could be recovered by other means. To this end, Arslanagić et al. [2] defined MSTs as the sub-class of session types without the ';' construct. They established a minimality result: every well-typed session process can be decomposed into a process typable with MSTs. Their result is inspired by Parrow's work on trios processes for the $\pi$-calculus [12]. The minimality result justifies session types in terms of themselves, and shows that sequentiality in types is useful but not indispensable, because it can be precisely mimicked by the process decomposition.

The minimality result in [2] holds for a higher-order process calculus called $\mathrm{HO}$, in which values are only abstractions (functions from names to processes). HO does not include name passing nor process recursion, but it can encode them precisely [9, 11]. An important question left open in [2] is whether the minimality result holds for the session $\pi$-calculus (dubbed $\pi$ ), the language in which values are names and for which session types have been more widely studied from foundational and practical perspectives.

In this paper, we positively answer this question. Our approach is simple, perhaps even deceptively so. In order to establish the minimality result for $\pi$, we compose the decomposition in [2] with the mutual encodings between $\mathrm{HO}$ and $\pi$ given in $[9,11]$.

Let $\mu \pi$ and $\mu \mathrm{HO}$ denote the process languages $\pi$ and $\mathrm{HO}$ with MSTs (rather than with standard session types). Also, let $\mathcal{D}(\cdot)$ denote the decomposition function from $\mathrm{HO}$ to $\mu \mathrm{HO}$ defined in [2]. Kouzapas et al. $[9,11]$ gave typed encodings from $\pi$ to $\mathrm{HO}$ (denoted $\llbracket \cdot \rrbracket_{g}^{1}$ ) and from $\mathrm{HO}$ to $\pi$ (denoted $\llbracket \cdot \rrbracket^{2}$ ). Therefore, given $\mathcal{D}(\cdot)$, $\llbracket \cdot \rrbracket_{g}^{1}$, and

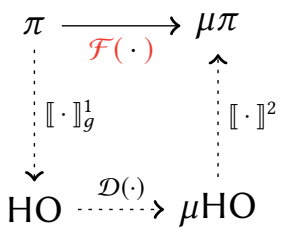

Figure 1: Decomposition by composition.

to follow Figure 1. This is sound for our purposes, because $\llbracket \cdot \rrbracket_{g}^{1}$ and $\llbracket \cdot \rrbracket^{2}$ preserve sequentiality in processes and their types.

The first contribution of this paper is the decomposition function $\mathcal{F}(\cdot)$, whose correctness follows from that of its constituent functions. $\mathcal{F}(\cdot)$ is significant, as it provides an elegant, positive answer to the question of whether the minimality result in [2] holds for $\pi$. Indeed, it proves that the values exchanged do not influence sequentiality in session types: the minimality result of [2] is not specific to the abstraction-passing language $\mathrm{HO}$.

However, $\mathcal{F}(\cdot)$ is not entirely satisfactory. A side effect of composing $\mathcal{D}(\cdot)$, $\llbracket \cdot \rrbracket_{g}^{1}$, and $\llbracket \cdot \rrbracket^{2}$ is that the resulting decomposition of $\pi$ into $\mu \pi$ is inefficient, as it includes redundant synchronizations. These shortcomings are particularly noticeable in the treatment of recursive processes. The second contribution of this paper is an optimized variant of $\mathcal{F}(\cdot)$, dubbed $\mathcal{F}^{*}(\cdot)$, in which we remove redundant synchronizations and target recursive processes and 


$$
\begin{aligned}
n & ::=a, b \mid s, \bar{s} \\
u, w & ::=n \mid x, y, z \\
V, W: & :=u|\lambda x . P| x, y, z \\
P, Q & ::=u !\langle V\rangle . P \mid u ?(x) . P \\
& |V u| P|Q|(v n) P|0| X \mid \mu X . P
\end{aligned}
$$

Figure 2: Syntax of $\mathrm{HO} \pi$. The sub-language $\mathrm{HO}$ lacks shaded constructs, while $\pi$ lacks boxed constructs.

variables directly, exploiting the fact that $\pi$ supports recursion natively.

Contributions. The main contributions of this paper are:

(1) A minimality result for $\pi$, based on the function $\mathcal{F}(\cdot)$.

(2) $\mathcal{F}^{*}(\cdot)$, an optimized variant of $\mathcal{F}(\cdot)$, without redundant communications and with native support for recursion.

(3) Examples for $\mathcal{F}(\cdot)$ and $\mathcal{F}^{*}(\cdot)$.

Due to space limits, omitted material (definitions, correctness proofs for $\mathcal{F}(\cdot)$ and $\mathcal{F}^{*}(\cdot)$, additional examples) can be found in [1] Throughout the paper, we use red and blue colors to distinguish elements of the first and second decompositions, respectively.

\section{PRELIMINARIES}

$\mathrm{HO}$ and $\pi$ are actually sub-languages of $\mathrm{HO} \pi[10,11]$, for which we recall its syntax, semantics, and session type system. We also recall the mutual encodings between $\mathrm{HO}$ and $\pi[9,11]$. Finally, we briefly discuss MSTs for $\mathrm{HO}$, and the minimality result in [2].

\section{1 $\mathrm{HO} \pi$ (and its Sub-languages $\mathrm{HO}$ and $\pi$ )}

Fig. 2 gives the syntax of processes $P, Q, \ldots$, values $V, W, \ldots$, and conventions for names. Identifiers $a, b, c, \ldots$ denote shared names, while $s, \bar{s}, \ldots$ are used for session names. Duality is defined only on session names, thus $\overline{\bar{s}}=s$, but $\bar{a}=a$. Names (shared or sessions) are denoted by $m, n \ldots$, and $x, y, z, \ldots$ range over variables. We write $\widetilde{x}$ to denote a tuple $\left(x_{1}, \ldots, x_{n}\right)$, and use $\epsilon$ to denote the empty tuple.

An abstraction $\lambda x . P$ binds $x$ to its body $P$. In processes, sequencing is specified via prefixes. The output prefix, $u !\langle V\rangle . P$, sends value $V$ on name $u$, then continues as $P$. Its dual is the input prefix, $u$ ? $(x) . P$, in which variable $x$ is bound. Parallel composition, $P \mid Q$, reflects the combined behaviour of two processes running simultaneously. Restriction $(v n) P$ binds the endpoints $n, \bar{n}$ in process $P$. Process 0 denotes inaction. Recursive variables and recursive processes are denoted $X$ and $\mu X . P$, respectively. Replication is denoted by the shorthand notation $* P$, which stands for $\mu X .(P \mid X)$.

The sets of free variables, sessions, and names of a process are denoted $f v(P), f s(P)$, and $f n(P)$. A process $P$ is closed if $f v(P)=\emptyset$. We write $u !\langle\rangle . P$ and $u$ ?().P when the communication objects are not relevant. Also, we omit trailing occurrences of $\mathbf{0}$.

As Fig. 2 details, the sub-languages $\pi$ and $\mathrm{HO}$ of $\mathrm{HO} \pi$ differ as follows: application $V u$ is only present in $\mathrm{HO}$; constructs for recursion $\mu X . P$ are present in $\pi$ but not in $\mathrm{HO}$.

$$
\begin{aligned}
& (\lambda x . P) u \longrightarrow P\{u / x\} \\
& n !\langle V\rangle . P|\bar{n} ?(x) \cdot Q \longrightarrow P| Q\{V / x\} \\
& \text { [App] } \\
& P \longrightarrow P^{\prime} \Rightarrow(v n) P \longrightarrow(v n) P^{\prime} \\
& P \longrightarrow P^{\prime} \Rightarrow P\left|Q \longrightarrow P^{\prime}\right| Q \\
& P \equiv Q \longrightarrow Q^{\prime} \equiv P^{\prime} \Rightarrow P \longrightarrow P^{\prime} \\
& P_{1}\left|P_{2} \equiv P_{2}\right| P_{1} \quad P_{1}\left|\left(P_{2} \mid P_{3}\right) \equiv\left(P_{1} \mid P_{2}\right)\right| P_{3} \\
& P|\mathbf{0} \equiv P \quad P|(v n) Q \equiv(v n)(P \mid Q)(n \notin \mathrm{fn}(P)) \\
& (v n) \mathbf{0} \equiv \mathbf{0} \quad \mu X . P \equiv P\{\mu X . P / X\} \quad P \equiv Q \text { if } P \equiv_{\alpha} Q
\end{aligned}
$$

Figure 3: Operational Semantics of $\mathrm{HO} \pi$.

$$
\begin{array}{rlrl}
U::=C \mid L & C::=S|\langle S\rangle|\langle L\rangle \\
L::=U \rightarrow \diamond \mid U \rightarrow \diamond & S::=!\langle U\rangle ; S \mid ?(U) ; S \\
& |\mu \mathrm{t} . S| \mathrm{t} \mid \text { end }
\end{array}
$$

$$
\begin{array}{lll}
U::=\widetilde{C} \rightarrow \diamond \mid \widetilde{C} \multimap \diamond & C::=M \mid\langle U\rangle \\
\gamma::=\text { end } \mid \mathrm{t} & M::=\gamma|!\langle\widetilde{U}\rangle ; \gamma| ?(\widetilde{U}) ; \gamma \mid \mu \mathrm{t} . M
\end{array}
$$

Figure 4: STs for $\mathrm{HO} \pi$ (top) and MSTs for $\mathrm{HO}$ (bottom).

The operational semantics of $\mathrm{HO} \pi$, enclosed in Figure 3, is expressed through a reduction relation, denoted $\longrightarrow$. Reduction is closed under structural congruence, $\equiv$, which identifies equivalent processes from a structural perspective. We write $P\{V / x\}$ to denote the capture-avoiding substitution of variable $x$ with value $V$ in $P$. We write ' \{\} ' to denote the empty substitution. In Figure 3, Rule [App] denotes application, which only concerns names. Rule [Pass] defines a shared or session interaction on channel $n$ 's endpoints. The remaining rules are standard.

\subsection{Session Types for $\mathrm{HO} \pi$}

Fig. 4 (top) gives the syntax of types. Value types $U$ include the first-order types $C$ and the higher-order types $L$. Session types are denoted with $S$ and shared types with $\langle S\rangle$ and $\langle L\rangle$. We write $\diamond$ to denote the process type. The functional types $U \rightarrow \diamond$ and $U \multimap$ $\diamond$ denote shared and linear higher-order types, respectively. The output type ! $\langle U\rangle ; S$ is assigned to a name that first sends a value of type $U$ and then follows the type described by $S$. Dually, ?(U); $S$ denotes an input type. Type end is the termination type. We assume the recursive type $\mu \mathrm{t} . S$ is guarded, i.e., the type variable $\mathrm{t}$ only appears under prefixes. This way, e.g., the type $\mu$ t.t is not allowed. The sets of free/bound variables of a type $S$ are defined as usual; the sole binder is $\mu \mathrm{t}$.S. Closed session types do not have free type variables.

Session types for $\mathrm{HO}$ exclude $C$ from value types $U$; session types for $\pi$ exclude $L$ from value types $U$ and $\langle L\rangle$ from $C$.

We write $S_{1}$ dual $S_{2}$ if $S_{1}$ is the dual of $S_{2}$. Intuitively, duality converts ! into ? (and vice-versa). This intuitive definition is enough for our purposes; the formal definition is co-inductive, see $[10,11]$. 
Typing environments are defined below:

$$
\begin{array}{ccc}
\Gamma::=\emptyset|\Gamma, x: U \rightarrow \diamond| \Gamma, u:\langle S\rangle|\Gamma, u:\langle L\rangle| \Gamma, X: \Delta \\
\Lambda::=\emptyset \mid \Lambda, x: U \multimap \diamond & \Delta::=\emptyset \mid \Delta, u: S
\end{array}
$$

$\Gamma, \Lambda$, and $\Delta$ satisfy different structural principles. $\Gamma$ maps variables and shared names to value types, and recursive variables to session environments; it admits weakening, contraction, and exchange principles. While $\Lambda$ maps variables to linear higher-order types, $\Delta$ maps session names to session types. Both $\Lambda$ and $\Delta$ are only subject to exchange. The domains of $\Gamma, \Lambda$ and $\Delta$ (denoted $\operatorname{dom}(\Gamma), \operatorname{dom}(\Lambda)$, and $\operatorname{dom}(\Delta))$ are assumed pairwise distinct.

Given $\Gamma$, we write $\Gamma \backslash x$ to denote the environment obtained from $\Gamma$ by removing the assignment $x: U \rightarrow \diamond$, for some $U$. This notation applies similarly to $\Delta$ and $\Lambda$; we write $\Delta \backslash \Delta^{\prime}$ (and $\Lambda \backslash \Lambda^{\prime}$ ) with the expected meaning. Notation $\Delta_{1} \cdot \Delta_{2}$ means the disjoint union of $\Delta_{1}$ and $\Delta_{2}$. We define typing judgements for values $V$ and processes $P$ :

$$
\Gamma ; \Lambda ; \Delta \vdash V \triangleright U \quad \Gamma ; \Lambda ; \Delta \vdash P \triangleright \diamond
$$

The judgement on the left says that under environments $\Gamma, \Lambda$, and $\Delta$ value $V$ has type $U$; the judgement on the right says that under environments $\Gamma, \Lambda$, and $\Delta$ process $P$ has the process type $\diamond$. The typing rules are presented in [1].

Type soundness for $\mathrm{HO} \pi$ relies on two auxiliary notions:

Definition 2.1 (Session Environments: Balanced/Reduction). Let $\Delta$ be a session environment.

- $\Delta$ is balanced if whenever $s: S_{1}, \bar{s}: S_{2} \in \Delta$ then $S_{1}$ dual $S_{2}$.

- We define reduction $\longrightarrow$ on session environments as:

$$
\Delta, s: !\langle U\rangle ; S_{1}, \bar{s}: ?(U) ; S_{2} \longrightarrow \Delta, s: S_{1}, \bar{s}: S_{2}
$$

Theorem 2.2 (Type Soundness [10]). Suppose $\Gamma ; \emptyset ; \Delta \vdash P \triangleright \diamond$ with $\Delta$ balanced. Then $P \longrightarrow P^{\prime}$ implies $\Gamma ; \emptyset ; \Delta^{\prime} \vdash P^{\prime} \triangleright \diamond$ and $\Delta=\Delta^{\prime}$ or $\Delta \longrightarrow \Delta^{\prime}$ with $\Delta^{\prime}$ balanced.

\subsection{Mutual Encodings between $\pi$ and $\mathrm{HO}$}

The encodings $\llbracket \cdot \rrbracket_{g}^{1}: \pi \rightarrow \mathrm{HO}$ and $\llbracket \cdot \rrbracket^{2}: \mathrm{HO} \rightarrow \pi$ are typed: each consists of a translation on processes and a translation on types. This way, $\boldsymbol{\gamma} \cdot\rangle^{1}$ translates types for first-order processes into types for higher-order processes, while $(\cdot)^{2}$ operates in the opposite direction-see Figures 5 and 6, respectively. Remarkably, these translations on processes and types do not alter their sequentiality.

From $\pi$ to HO. To mimic the sending of name $w$, the encoding $\llbracket \cdot \rrbracket_{g}^{1}$ encloses $w$ within the body of an input-guarded abstraction. The corresponding input process receives this higher-order value, applies it on a restricted session, and sends the encoded continuation through the session's dual.

Several auxiliary notions are used to encode recursive processes; we describe them intuitively (see [11] for full details). The key idea is to encode recursive processes in $\pi$ using a "duplicator" process in $\mathrm{HO}$, circumventing linearity by replacing free names with variables. The parameter $g$ is a map from process variables to sequences of name variables. Also, $\|\cdot\|$ maps sequences of session names into sequences of variables, and $\|\cdot\|_{\emptyset}$ maps processes with free names to processes without free names (but with free variables instead).

The encoding $(\cdot\rangle^{1}$ depends on the auxiliary function $\lfloor\cdot\rfloor^{1}$, defined on value types. Following the encoding on processes, this mapping on values takes a first-order value type and encodes it
Terms:

$$
\begin{aligned}
& \llbracket u !\langle w\rangle . P \rrbracket_{g}^{1} \stackrel{\text { def }}{=} u !\langle\lambda z . z ?(x) \cdot(x w)\rangle \cdot \llbracket P \rrbracket_{g}^{1} \\
& \llbracket u ?(x: C) \cdot Q \rrbracket_{g}^{1} \stackrel{\text { def }}{=} u ?(y) \cdot(v s)\left(y s \mid \bar{s} !\left\langle\lambda x . \llbracket Q \rrbracket_{g}^{1}\right\rangle .0\right) \\
& \llbracket P\left|Q \rrbracket_{g}^{1} \stackrel{\text { def }}{=} \llbracket P \rrbracket_{g}^{1}\right| \llbracket Q \rrbracket_{g}^{1} \\
& \llbracket(v n) P \rrbracket_{g}^{1} \stackrel{\text { def }}{=}(v n) \llbracket P \rrbracket_{g}^{1} \\
& \llbracket 0 \rrbracket_{g}^{1} \stackrel{\text { def }}{=} 0 \\
& \llbracket \mu X . P \rrbracket_{g}^{1} \stackrel{\text { def }}{=}(v s)\left(\bar{s} !\langle V\rangle .0 \mid s ?\left(z_{X}\right) . \llbracket P \rrbracket_{g,\{X \rightarrow \tilde{n}\}}^{1}\right) \\
& \text { where }(\tilde{n}=\mathrm{fn}(P)) \\
& V=\lambda(\|\tilde{n}\|, y) \cdot y ?\left(z_{X}\right) \cdot\left\|\llbracket P \rrbracket_{g,\{X \rightarrow \tilde{n}\}}^{1}\right\|_{\emptyset} \\
& \llbracket X \rrbracket_{g}^{1} \stackrel{\text { def }}{=}(v s)\left(z_{X}(\tilde{n}, s) \mid \bar{s} !\left\langle z_{X}\right\rangle .0\right) \quad(\tilde{n}=g(X)) \\
& \text { Types: } \\
& \lfloor S\rfloor^{1 \stackrel{\text { def }}{=}}\left(?\left(\langle S\rangle^{1} \multimap \diamond\right) \text {; end }\right) \multimap \diamond \\
& \lfloor\langle S\rangle\rfloor \stackrel{1}{1 \text { def }}=\left(?\left(\left\langle\langle S\rangle^{1}\right\rangle \rightarrow \diamond\right) \text {; end }\right) \multimap \diamond \\
& \langle!\langle U\rangle ; S\rangle^{1} \stackrel{\text { def }}{=} !\left\langle\lfloor U\rfloor^{1}\right\rangle ;\langle S\rangle^{1} \\
& \langle ?(U) ; S\rangle^{1} \stackrel{\text { def }}{=} ?\left(\lfloor U\rfloor^{1}\right) ;\langle S\rangle^{1} \\
& \langle\langle S\rangle\rangle^{1} \stackrel{\text { def }}{=}\left\langle\langle S\rangle^{1}\right\rangle \quad\langle\mu \mathrm{t} . S\rangle^{1} \stackrel{\text { def }}{=} \mu \mathrm{t} .\langle S\rangle^{1} \\
& \langle\text { end }\rangle^{1} \stackrel{\text { def }}{=} \text { end } \quad\langle t\rangle^{1} \stackrel{\text { def }}{=} \mathrm{t}
\end{aligned}
$$

Figure 5: Typed encoding of $\pi$ into $\mathrm{HO}$, selection from [11]. Above, $f \mathrm{n}(P)$ is a lexicographically ordered sequence of free names in $P$. Maps $\|\cdot\|$ and $\|\cdot\|_{\sigma}$ can be found in [1].

into a linear higher-order value type, which encloses an input type that expects to receive another higher-order type. Notice how the innermost higher-order value type is either shared or linear, following the nature of the given type.

From $\mathrm{HO}$ to $\pi$. The encoding $\llbracket \cdot \rrbracket^{2}$ simulates higher-order communication using first-order constructs, following Sangiorgi [13]. The idea is to use trigger names, which point towards copies of inputguarded server processes that should be activated. The encoding of abstraction sending distinguishes two cases: if the abstraction body does not contain any free session names (which are linear), then the server can be replicated. Otherwise, if the value contains session names then its corresponding server name must be used exactly once. The encoding of abstraction receiving proceeds inductively, noticing that the variable is now a placeholder for a first-order name. The encoding of application is also in two cases; both of them depend on the creation of a fresh session, which is used to pass around the applied name.

\subsection{Minimal Session Types for $\mathrm{HO}$}

The syntax of MSTs for $\mathrm{HO}$ is in Fig. 4 (bottom). We write $\mu \mathrm{HO}$ to denote $\mathrm{HO}$ with MSTs. The decomposition $\mathcal{D}(\cdot)$ in [2] relies crucially on the ability of communicating tuples of values. Hence, value types are of the form $\widetilde{C} \rightarrow \diamond$ and $\widetilde{C} \multimap \diamond$. Similarly, minimal session 


$$
\begin{gathered}
\llbracket u !\langle\lambda x \cdot Q\rangle \cdot P \rrbracket^{2} \stackrel{\text { def }}{=} \\
\begin{cases}(v a)\left(u !\langle a\rangle \cdot\left(\llbracket P \rrbracket^{2} \mid * a ?(y) \cdot y ?(x) \cdot \llbracket Q \rrbracket^{2}\right)\right) & \text { if } f s(Q)=\emptyset \\
(v a)\left(u !\langle a\rangle \cdot\left(\llbracket P \rrbracket^{2} \mid a ?(y) \cdot y ?(x) \cdot \llbracket Q \rrbracket^{2}\right)\right) & \text { otherwise }\end{cases} \\
\llbracket u ?(x) \cdot P \rrbracket^{2} \stackrel{\text { def }}{=} u ?(x) \cdot \llbracket P \rrbracket^{2} \\
\llbracket x u \rrbracket^{2} \stackrel{\text { def }}{=}(v s)(x !\langle s\rangle \cdot \bar{s} !\langle u\rangle \cdot 0) \\
\llbracket(\lambda x . P) u \rrbracket^{2} \stackrel{\text { def }}{=}(v s)\left(s ?(x) \cdot \llbracket P \rrbracket^{2} \mid \bar{s} !\langle u\rangle \cdot 0\right) \\
\quad \text { Types: } \\
\left\langle!\langle S \multimap \diamond\rangle ; S_{1}\right\rangle^{2} \stackrel{\text { def }}{=} !\left\langle\left\langle ?\left(\langle S\rangle^{2}\right) ; \text { end }\right\rangle\right\rangle ;\left\langle S_{1}\right\rangle^{2} \\
\left.\quad ?(S \multimap \diamond) ; S_{1}\right\rangle^{2} \stackrel{\text { def }}{=} ?\left(\left\langle ?\left(\langle S\rangle^{2}\right) ; \text { end }\right\rangle\right) ;\left\langle S_{1}\right\rangle^{2}
\end{gathered}
$$

Figure 6: Typed encoding of HO into $\pi$ [11].

types for output and input are of the form ! $\langle\widetilde{U}\rangle$; end and ? $(\widetilde{U})$; end: they communicate tuples of values but lack a continuation.

Following Parrow [12], $\mathcal{D}(\cdot)$ is defined in terms of a breakdown function $\mathcal{B}_{\tilde{x}}^{k}(\cdot)$, which translates a process into a composition of trios processes (or simply trios). A trio is a process with exactly three nested prefixes. If $P$ is a sequential process with $k$ nested actions, then $\mathcal{D}(P)$ will contain $k$ trios running in parallel: each trio in $\mathcal{D}(P)$ will enact exactly one prefix from $P$; the breakdown function must be carefully designed to ensure that trios trigger each other in such a way that $\mathcal{D}(P)$ preserves the prefix sequencing in $P$. While trios decompositions elegantly induce processes typable with MSTs, they are not goal in themselves; rather, they offer one possible path to better understand sequentiality in session types.

We use some useful terminology for trios [12]. The context of a trio is a tuple of variables $\widetilde{x}$, possibly empty, which makes variable bindings explicit. We use a reserved set of propagator names (or simply propagators), denoted by $c_{k}, c_{k+1}, \ldots$, to carry contexts and trigger the subsequent trio. Propagators with recursive types are denoted by $c_{k}^{r}, c_{k+1}^{r}, \ldots$ We say that a leading trio is the one that receives a context, performs an action, and triggers the next trio; a control trio only activates other trios.

The breakdown function works on both processes and values. The breakdown of process $P$ is denoted by $\mathcal{B}_{\tilde{x}}^{k}(P)$, where $k$ is the index for the propagators $c_{k}$, and $\tilde{x}$ is the context to be received by the previous trio. Similarly, the breakdown of a value $V$ is denoted by $\mathcal{V}_{\tilde{x}}^{k}(V)$. Table 1 gives the breakdown function defined in [2] for the sub-language of $\mathrm{HO}$ without recursion-this is the so-called core fragment. In the figure, we include side conditions that use the one-line conditional $x=(c)$ ? $s_{1}: s_{2}$ to express that $x=s_{1}$ if condition $c$ is true, and $x=s_{2}$ otherwise. Notice that for session types we have either $C=S$ or $C=\langle S\rangle$.

To formally define $\mathcal{D}(\cdot)$ in terms of $\mathcal{B}_{\tilde{x}}^{k}(\cdot)$, we need some notation. Let $\widetilde{u}=\left(a, b, s, s^{\prime}, \ldots\right)$ be a finite tuple of names. We shall write $\operatorname{init}(\widetilde{u})$ to denote the tuple $\left(a_{1}, b_{1}, s_{1}, s_{1}^{\prime}, \ldots\right)$. We say that a process has been initialized if all of its names have some index.

Definition 2.3 (Decomposing Processes [2]). Let $P$ be a closed HO process such that $\widetilde{u}=\mathrm{fn}(P)$. The decomposition of $P$, denoted $\mathcal{D}(P)$,

$$
\begin{aligned}
& \mathcal{G}(!\langle U\rangle ; S)= \begin{cases}!\langle\mathcal{G}(U)\rangle ; \text { end } & \text { if } S=\text { end } \\
!\langle\mathcal{G}(U)\rangle ; \text { end }, \mathcal{G}(S) & \text { otherwise }\end{cases} \\
& \mathcal{G}(?(U) ; S)= \begin{cases}?(\mathcal{G}(U)) ; \text { end } & \text { if } S=\text { end } \\
?(\mathcal{G}(U)) ; \text { end }, \mathcal{G}(S) & \text { otherwise }\end{cases} \\
& \mathcal{G}(C \multimap \diamond)=\mathcal{G}(C) \multimap \diamond \quad \mathcal{G}(\text { end })=\text { end } \\
& \mathcal{G}(C \rightarrow \diamond)=\mathcal{G}(C) \rightarrow \diamond \quad \mathcal{G}(\langle U\rangle)=\langle\mathcal{G}(U)\rangle \\
& \mathcal{G}\left(S_{1}, \ldots, S_{n}\right)=\mathcal{G}\left(S_{1}\right), \ldots, \mathcal{G}\left(S_{n}\right)
\end{aligned}
$$

Figure 7: Decomposition of types (cf. Def. 2.4)

is defined as:

$$
\mathcal{D}(P)=(v \widetilde{c})\left(\overline{c_{k}} !\langle\rangle .0 \mid \mathcal{B}_{\epsilon}^{k}(P \sigma)\right)
$$

where: $k>0 ; \tilde{c}=\left(c_{k}, \ldots, c_{k+|P|-1}\right) ; \sigma=\{\operatorname{init}(\tilde{u}) / \tilde{u}\}$; and the breakdown function $\mathcal{B}_{\tilde{x}}^{k}(\cdot)$, is as defined in Table 1 .

Definition 2.4 (Decomposing Session Types). The decomposition function on the types of Fig. 4, denoted $\mathcal{G}(\cdot)$, is defined in Fig. 7.

As already mentioned, the minimality result in [2] is that if $P$ is a well-typed $\mathrm{HO}$ process then $\mathcal{D}(P)$ is a well-typed $\mu \mathrm{HO}$ process. It attests that the sequentiality in the session types for $P$ is appropriately accommodated by the decomposition $\mathcal{D}(P)$. Its proof relies on an auxiliary result establishing the typability of $\mathcal{B}_{\tilde{x}}^{k}(P)$.

Theorem 2.5 (Minimality Result [2]). Let $P$ be a closed $\mathrm{HO}$ process with $\widetilde{u}=\mathrm{fn}(P)$ and $\sigma=\{\operatorname{init}(\widetilde{u}) / \widetilde{u}\}$. If $\Gamma ; \emptyset ; \Delta \vdash P \triangleright \diamond$ then

$$
\mathcal{G}(\Gamma \sigma) ; \emptyset ; \mathcal{G}(\Delta \sigma) \vdash \mathcal{D}(P) \triangleright \diamond
$$

Having summarized the results on which our developments stand, we now move on to establish the minimality result but for $\pi$.

\section{DECOMPOSE BY COMPOSITION}

We define a decomposition function $\mathcal{F}(\cdot): \pi \rightarrow \mu \pi$, given in terms of a breakdown function denoted $\mathcal{A}_{\tilde{x}}^{k}(\cdot) g$ (cf. Tab. 2). Following Figure 1, this breakdown function will result from the composition of $\llbracket \cdot \rrbracket_{g}^{1}, \mathcal{B}_{\tilde{x}}^{k}(\cdot)$, and $\llbracket \cdot \rrbracket^{2}$, i.e., $\mathcal{A}_{\tilde{x}}^{k}(\cdot)_{g}=\llbracket \mathcal{B}_{\tilde{x}}^{k}\left(\llbracket \cdot \rrbracket_{g}^{1}\right) \rrbracket^{2}$. Using $\mathcal{F}(\cdot)$, we obtain a minimality result for $\pi$, given by Theorem 3.11 .

\subsection{Key Idea}

Conceptually, $\mathcal{F}(\cdot)$ can be obtained in two steps: first, the composition $\mathcal{B}_{\tilde{x}}^{k}\left(\llbracket \cdot \rrbracket_{g}^{1}\right)$, which returns a process in $\mu \mathrm{HO}$; second, a step that transforms that $\mu \mathrm{HO}$ process into a $\mu \pi$ process using $\llbracket \cdot \rrbracket^{2}$. We illustrate these two steps for output and input processes.

Output Let us write $\mathcal{A}_{\tilde{x}}^{\prime k}(\cdot)_{g}$ to denote the (partial) composition involved in the first step. Given $P=u_{i} !\left\langle w_{j}\right\rangle$.Q, we first obtain:

$$
\mathcal{A}_{\tilde{x}}^{\prime k}\left(u_{i} !\left\langle w_{j}\right\rangle \cdot Q\right)_{g}=c_{k} ?(\tilde{x}) \cdot u_{i} !\langle W\rangle \cdot \overline{c_{k+3}} !\langle\tilde{x}\rangle \mid \mathcal{A}_{\tilde{x}}^{\prime k+3}(Q \sigma)_{g}
$$

where $\sigma=\left(u_{i}: S\right) ?\left\{u_{i+1} / u_{i}\right\}:\{\}$ and

$$
W=\lambda z_{1} \cdot\left(\overline{c_{k+1}} !\langle\rangle\left|c_{k+1} ?() \cdot z_{1} ?(x) \cdot \overline{c_{k+2}} !\langle x\rangle\right| c_{k+2} ?(x) \cdot(x \widetilde{w})\right)
$$




\begin{tabular}{|c|c|c|}
\hline$P$ & $\mathcal{B}_{\tilde{x}}^{k}(P)$ & \\
\hline$u_{i} !\langle V\rangle \cdot Q$ & $c_{k} ?(\widetilde{x}) \cdot u_{i} !\langle\widehat{V}\rangle \cdot \overline{c_{k+l+1}} !\langle\widehat{z}\rangle \mid \mathcal{B}_{\tilde{z}}^{k+l+1}(Q \sigma)$ & $\begin{array}{l}\widehat{V}=\mathcal{V}_{\hat{y}}^{k+1}(V \sigma) \\
\widetilde{y}=f v(V) \\
l=|V|\end{array}$ \\
\hline$u_{i} ?(y) \cdot Q$ & $c_{k} ?(\widetilde{x}) \cdot u_{i} ?(y) \cdot \overline{c_{k+1}} !\left\langle\widetilde{x}^{\prime}\right\rangle \mid \mathcal{B}_{\tilde{x}^{\prime}}^{k+1}(Q \sigma)$ & $\begin{array}{l}\widetilde{x}^{\prime}=f v(Q) \\
\sigma=\operatorname{next}\left(u_{i}\right)\end{array}$ \\
\hline$V u_{i}$ & $c_{k} ?(\widetilde{x}) \cdot \mathcal{V}_{\tilde{x}}^{k+1}(V) \widetilde{m}$ & $\begin{array}{ll}u_{i}: C & \widetilde{m}=\left(u_{i}, \ldots, u_{i+\mid} \mathcal{G}(C) \mid-1\right) \\
\widetilde{x}=f v(V) & \end{array}$ \\
\hline$(v s: C) P^{\prime}$ & $(v \widetilde{s}: \mathcal{G}(C)) \mathcal{B}_{\tilde{x}}^{k}\left(P^{\prime} \sigma\right)$ & $\begin{array}{l}\widetilde{s}=\left(s_{1}, \ldots, s_{|\mathcal{G}(C)|}\right) \\
\sigma=(C=S) ?\left\{s_{1} \bar{s}_{1} / s \bar{s}\right\}:\left\{s_{1} / s\right\} \quad \widetilde{x}=f v\left(P^{\prime}\right)\end{array}$ \\
\hline$Q \mid R$ & $c_{k} ?(\widetilde{x}) \cdot \overline{c_{k+1}} !\langle\widetilde{y}\rangle \cdot \overline{c_{k+l+1}} !\langle\bar{z}\rangle\left|\mathcal{B}_{\tilde{y}}^{k+1}(Q)\right| \mathcal{B}_{\tilde{z}}^{k+l+1}(R)$ & $\widetilde{y}=f v(Q) \quad \tilde{z}=f v(R) \quad l=|Q|$ \\
\hline 0 & $c_{k} ?() .0$ & \\
\hline$V$ & $\mathcal{V}_{\tilde{x}}^{k}(V)$ & \\
\hline$y$ & $y$ & \\
\hline$\lambda u: C^{\sim} . P$ & $\lambda \widetilde{y} \cdot(v \widetilde{c})\left(\overline{c_{k}} !\langle\widetilde{x}\rangle \mid \mathcal{B}_{\tilde{x}}^{k}\left(P\left\{y_{1} / y\right\}\right)\right)$ & $\begin{aligned} \widetilde{x} & =\mathrm{fv}(V) \\
\widetilde{y} & =\left(y_{1}, \ldots, y|\mathcal{G}(C)|\right) \\
\widetilde{c} & =(\sim=\rightarrow) ?\left(c_{k}, \ldots, c_{k+|P|-1}\right): \epsilon\end{aligned}$ \\
\hline
\end{tabular}

Table 1: The breakdown function for $\mathrm{HO}$ processes and values (core fragment from [2]).

We have that $\mathcal{A}^{\prime}{ }_{\tilde{x}}\left(u_{i} !\left\langle w_{j}\right\rangle \cdot Q\right)_{g}$ is a process in $\mu \mathrm{HO}$. The second step uses $\llbracket \cdot \rrbracket^{2}$ to convert it into the following $\mu \pi$ process:

$$
\begin{gathered}
c_{k} ?(\widetilde{x}) \cdot(v a)\left(u _ { i } ! \langle a \rangle \cdot \left(\overline{c_{k+3}} !\langle\widetilde{x}\rangle\left|\mathcal{A}_{\tilde{x}}^{k+3}(Q \sigma)_{g}\right|\right.\right. \\
a ?(y) \cdot y ?\left(z_{1}\right) \cdot \overline{c_{k+1}} !\left\langle z_{1}\right\rangle\left|c_{k+1} ?\left(z_{1}\right) \cdot z_{1} ?(x) \cdot \overline{c_{k+2}} !\langle x\rangle\right| \\
\left.\left.c_{k+2} ?(x) \cdot(v s)(x !\langle s\rangle \cdot \bar{s} !\langle\widetilde{w}\rangle)\right)\right)
\end{gathered}
$$

The subprocess mimicking the output action on $u_{i}$ is guarded by an input on $c_{k}$. Then, the output of $w$ on $u_{i}$ action is delegated to a different channel through several redirections: first a private name $a$ is sent, then along $a$ name for $z_{1}$ is received and so on; until, finally, the breakdown of $w$ is sent on name $s_{1}$. These names are propagated through local trios. We can see that upon action on $u_{i}$ unmodified context $\tilde{x}$ is sent to breakdown of continuation $Q$.

We say names typed with tail-recursive type are recursive names. Another form of output is when both $u_{i}$ and $w_{j}$ are recursive. This case is similar to the one just discussed, and omitted from Tab. 2. Input The breakdown of $u_{i} ?(w) . Q$ as follows:

$$
\begin{aligned}
& c_{k} ?(\widetilde{x}) \cdot u_{i} ?(y) \cdot \overline{c_{k+1}} !\langle\widetilde{x}, y\rangle \mid \\
& \left(v s_{1}\right)\left(c_{k+1} ?(\widetilde{x}, y) \cdot \overline{c_{k+2}} !\langle y\rangle \cdot \overline{c_{k+3}} !\langle\widetilde{x}\rangle \mid\right. \\
& c_{k+2} ?(y) \cdot(v s)\left(y !\langle s\rangle \cdot \bar{s} !\left\langle s_{1}\right\rangle\right) \mid \\
& c_{k+3} ?(\widetilde{x}) \cdot(v a)\left(\overline { s _ { 1 } } ! \langle a \rangle \cdot \left(\overline{c_{k+l+4}} !\langle\rangle\left|c_{k+l+4} ?() \cdot 0\right|\right.\right. \\
& \left.\left.\left.a ?\left(y^{\prime}\right) \cdot y^{\prime} ?(\widetilde{w}) \cdot\left(\overline{c_{k+4}} !\langle\widetilde{x}\rangle \mid \mathcal{A}_{\widetilde{x}}^{k+4}\left(Q\left\{w_{1} / w\right\} \sigma\right)_{g}\right)\right)\right)\right)
\end{aligned}
$$

The activation on $c_{k}$ enables the input on $u_{i}$. After several redirections, the actual input of variables $\widetilde{w}$ is on a name received for $y^{\prime}$, which binds them in the decomposition of $Q$. Hence, context $\widetilde{x}$ does not get extended for an inductive call: it only gets extended locally (propagated by $c_{k+1}$ ). Indeed, in the core fragment, the context is always empty and propagators only enable subsequent actions. The

$$
\begin{aligned}
C & :=M \mid\langle M\rangle \\
\gamma & ::=\text { end } \mid \mathrm{t} \\
M & ::=\gamma|!\langle\widetilde{C}\rangle ; \gamma| ?(\widetilde{C}) ; \gamma \mid \mu \mathrm{t} . M
\end{aligned}
$$

Figure 8: Minimal Session Types for $\pi$ (cf. Definition 3.1)

context does play a role in breaking down recursion: variables $z_{X}$ (generated to encode recursion) get propagated as context.

\subsection{Formal Definition}

Definition 3.1 (Minimal Session Types, MSTs). Minimal session types for $\pi$ are defined in Figure 8.

The breakdown function $\mathcal{A}_{\tilde{x}}^{k}(\cdot)_{g}$ for all constructs of $\pi$ is given in Table 2, using the following definitions.

Definition 3.2 (Degree of a Process). The degree of a $\pi$ process $P$, denoted $\lfloor P\rceil$, is defined as:

$$
\begin{aligned}
& \left\lfloor u_{i} !\left\langle w_{j}\right\rangle \cdot Q\right\rceil=\lfloor Q\rceil+3 \quad\lfloor(\text { vs:S }) Q\rceil=\lfloor Q\rceil \quad\lfloor\mathbf{0}\rceil=1 \\
& \left\lfloor u_{i} ?(x: C) \cdot Q\right\rceil=\lfloor Q\rceil+5 \quad\lfloor Q \mid R\rceil=\lfloor Q\rceil+\lfloor R\rceil+1 \\
& \lfloor X\rceil=4 \quad\lfloor\mu X . Q\rceil=\lfloor Q\rceil+4
\end{aligned}
$$

Definition 3.3 (Predicates on Types and Names). Let $C$ be a session type. We write $\operatorname{tr}(C)$ to indicate that $C$ is a tail-recursive session type. Also, given $u: C$, we write $\operatorname{lin}(u)$ if $C=S$ and $\neg \operatorname{tr}(S)$.

Definition 3.4 (Subsequent index substitution). Let $n_{i}$ be an indexed name. We define $\operatorname{next}\left(n_{i}\right)=\left(\operatorname{lin}\left(n_{i}\right)\right) ?\left\{n_{i+1} / n_{i}\right\}:\{\}$.

We define how to obtain MSTs for $\pi$ from standard session types:

Definition 3.5 (Decomposing First-Order Types). The decomposition function $\mathcal{H}(\cdot)$ on finite types, obtained by combining the 


\begin{tabular}{|c|c|c|}
\hline$P$ & $\mathcal{A}_{\tilde{x}}^{k}(P)_{g}$ & \\
\hline$u_{i} !\left\langle w_{j}\right\rangle \cdot Q$ & $\begin{array}{c}c_{k} ?(\widetilde{x}) \cdot(v a)\left(u_{i} !\langle a\rangle \cdot\left(\overline{c_{k+3}} !\langle\widetilde{x}\rangle\left|\mathcal{A}_{\tilde{x}}^{k+3}(Q \sigma)_{g}\right|\right.\right. \\
a ?(y) \cdot y ?\left(z_{1}\right) \cdot \overline{c_{k+1}} !\left\langle z_{1}\right\rangle\left|c_{k+1} ?\left(z_{1}\right) \cdot z_{1} ?(x) \cdot \overline{c_{k+2}} !\langle x\rangle\right| \\
\left.\left.c_{k+2} ?(x) \cdot(v s)(x !\langle s\rangle \cdot \bar{s} !\langle\widetilde{w}\rangle)\right)\right)\end{array}$ & $\begin{array}{l}w_{j}: C \\
\widetilde{w}=\left(w_{j}, \ldots, w_{j+|\mathcal{H}(C)|-1}\right) \\
\sigma=\operatorname{next}\left(u_{i}\right)\end{array}$ \\
\hline$u_{i} ?(w) \cdot Q$ & 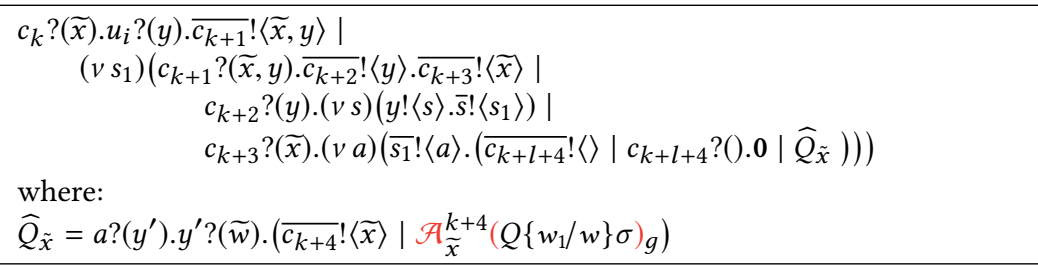 & $\begin{array}{l}w: C \\
\widetilde{w}=\left(w_{1}, \ldots, w_{|\mathcal{H}(C)|}\right) \\
l=\lfloor Q\rceil \\
\sigma=\operatorname{next}\left(u_{i}\right)\end{array}$ \\
\hline$r_{i} !\left\langle w_{j}\right\rangle \cdot P$ & $\begin{array}{l}c_{k} ?(\widetilde{x}) \cdot\left(v a_{1}\right) c^{r} !\left\langle a_{1}\right\rangle \cdot\left(\mathcal{A}_{\tilde{x}}^{k+3}(P)_{g} \mid a_{1} ?\left(y_{1}\right) \cdot y_{1} ?(\widetilde{z}) \cdot W\right) \\
\text { where: } \\
W=\left(v a_{2}\right)\left(z_{f(S)} !\left\langle a_{2}\right\rangle \cdot\left(\overline{c_{k+3}} !\langle\widetilde{x}\rangle \cdot c^{r} ?(b) \cdot(v s)(b !\langle s\rangle \cdot \bar{s} !\langle\widetilde{z}\rangle) \mid\right.\right. \\
\qquad \begin{aligned} a_{2} ?\left(y_{2}\right) \cdot y_{2} ?\left(z_{1}^{\prime}\right) \cdot\left(\overline{c_{k+1}} !\langle\rangle \mid\right. \\
\left.\left.\left.\left.\qquad c_{k+1} ?\right) \cdot z_{1}^{\prime} ?(x) \cdot \bar{c}_{k+2} !\langle x\rangle \mid c_{k+2} ?(x) \cdot\left(v s^{\prime}\right)\left(x !\left\langle s^{\prime}\right\rangle \cdot \bar{s}^{\prime} !\langle\widetilde{w}\rangle\right)\right)\right)\right)\end{aligned}\end{array}$ & $\begin{array}{l}r: S \wedge \operatorname{tr}(S) \\
\widetilde{z}=\left(z_{1}, \ldots, z_{\mid \mathcal{R}^{\prime \star}}(S) \mid\right. \\
\widetilde{c}=\left(c_{k+1}, c_{k+2}\right) \\
w: C \wedge \widetilde{w}=\left(w_{j}, \ldots, w_{j+|\mathcal{H}(C)|-1}\right)\end{array}$ \\
\hline$r_{i} ?(w) \cdot P$ & 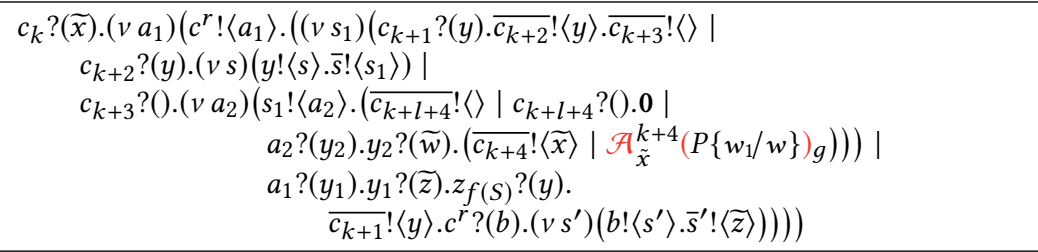 & $\begin{array}{l}r: S \wedge \operatorname{tr}(S) \\
\widetilde{z}=\left(z_{1}, \ldots, z_{\left|\mathcal{R}^{\prime \star}(S)\right|}\right) \\
l=\lfloor P\rceil \\
w: C \wedge \widetilde{w}=\left(w_{1}, \ldots, w_{|\mathcal{H}(C)|}\right)\end{array}$ \\
\hline$(v s: C) P^{\prime}$ & $(v \widetilde{s}: \mathcal{H}(C)) \mathcal{A}_{\tilde{x}}^{k}\left(P^{\prime} \sigma\right)_{g}$ & $\widetilde{s}=\left(s_{1}, \ldots, s_{|\mathcal{H}(C)|}\right) \quad \sigma=\left\{s_{1} \overline{s_{1}} / s \bar{s}\right\}$ \\
\hline$(v r: \mu \mathrm{t} . S) P^{\prime}$ & $\left(v \widetilde{r}: \mathcal{R}^{\prime}(S)\right) c^{r} ?(b) \cdot\left(v s^{\prime}\right)\left(b !\left\langle s^{\prime}\right\rangle \cdot \bar{s}^{\prime} !\langle\widetilde{r}\rangle\right)\left|c^{\bar{r}} ?(b) \cdot\left(v s^{\prime}\right)\left(b !\left\langle s^{\prime}\right\rangle \cdot \bar{s}^{\prime} !\langle\bar{r}\rangle\right)\right| \mathcal{A}_{\tilde{x}}^{k}\left(P^{\prime} \sigma\right)_{g}$ & $\begin{array}{ll}\operatorname{tr}(\mu \mathrm{t} . S) & \sigma=\left\{r_{1} \bar{r}_{1} / r \bar{r}\right\} \\
\widetilde{r}=\left(r_{1}, \ldots, r_{\left|\mathcal{R}^{\prime}(S)\right|}\right) & \\
\widetilde{\bar{r}}=\left(\bar{r}_{1}, \ldots, \bar{r}_{\left|\mathcal{R}^{\prime}(S)\right|}\right) & \\
\end{array}$ \\
\hline$Q_{1} \mid Q_{2}$ & $c_{k} ?(\tilde{x}) \cdot \overline{c_{k+1}} !\langle\tilde{y}\rangle \cdot \overline{c_{k+l+1}} !\langle\tilde{z}\rangle\left|\mathcal{A}_{\tilde{y}}^{k+1}\left(Q_{1}\right)_{g}\right| \mathcal{A}_{\tilde{z}}^{k+l+1}\left(Q_{2}\right)_{g}$ & $\widetilde{y}=f v\left(Q_{1}\right) \quad \widetilde{z}=f v\left(Q_{2}\right) \quad l=\lfloor Q\rceil$ \\
\hline 0 & $c_{k} ?() .0$ & \\
\hline$\mu X . P$ & 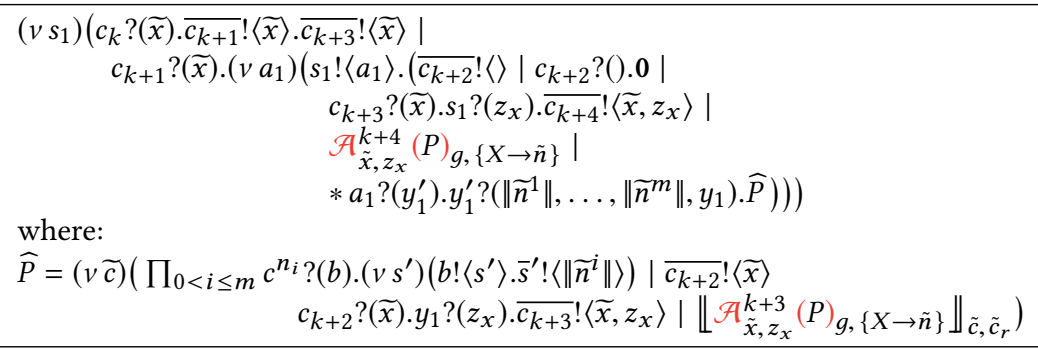 & $\begin{array}{l}\widetilde{n}=\mathrm{fn}(P) \\
m=|\widetilde{n}| \\
\|\widetilde{n}\|=\left(\left\|n_{1}\right\|, \ldots,\left\|n_{m}\right\|\right) \\
i \in\{1, \ldots, m\} . \\
\quad\left\|n_{i}\right\|: S_{i} \\
\quad\left\|\widetilde{n}^{i}\right\|=\left(\left\|n_{1}^{i}\right\|, \ldots,\left\|n_{\left|\mathcal{H}\left(S_{i}\right)\right|}^{i}\right\|\right) \\
\widetilde{c}=\left(c_{k+2}, \ldots, c_{k+\left\lfloor\|P\|_{g,\{X \rightarrow \tilde{n}\}}^{1}\right]+1}^{1}\right) \\
\widetilde{c}_{r}=\bigcup_{v \in \widetilde{n}} c^{v}\end{array}$ \\
\hline$X$ & $\begin{array}{l}\left(v s_{1}\right)\left(c_{k} ?\left(z_{x}\right) \cdot \overline{c_{k+1}} !\left\langle z_{x}\right\rangle \cdot \overline{c_{k+2}} !\left\langle z_{x}\right\rangle\left|c_{k+2} ?\left(z_{x}\right) \cdot \bar{s}_{1} !\left\langle z_{x}\right\rangle \cdot \overline{c_{k+3}} !\langle\rangle\right| c_{k+3} ?() \cdot 0\right. \\
\left.\quad c_{k+1} ?\left(z_{x}\right) \cdot\left(v a_{1}\right)\left(c^{n_{1}} !\left\langle a_{1}\right\rangle \cdot\left(a_{1} ?\left(y_{1}\right) \cdot y_{1} ? ?\left(\bar{z}_{1}\right) \ldots\left(v a_{j}\right) Q\right)\right)\right) \\
\text { where: } \quad\left(c^{n_{j}} !\left\langle a_{j}\right\rangle \cdot\left(a_{j} ?\left(y_{j}\right) \cdot y_{j} ?\left(\widetilde{z}_{j}\right) \cdot\left(v s^{\prime}\right)\left(z_{x} !\left\langle s^{\prime}\right\rangle \cdot \bar{s}^{\prime} !\left\langle\widetilde{z}_{1}, \ldots, \widetilde{z}_{j}, s_{1}\right\rangle\right)\right)\right) \\
Q\end{array}$ & $\begin{array}{l}\widetilde{n}=g(X) \\
|\widetilde{n}|=j \\
i \in\{1, \ldots, j\} \\
\quad n_{i}: S \wedge \operatorname{tr}\left(S_{i}\right) \\
\quad \widetilde{z_{i}}=\left(z_{1}^{i}, \ldots, z_{\left|\mathcal{R}^{\prime \star}\left(S_{i}\right)\right|}^{i}\right)\end{array}$ \\
\hline
\end{tabular}

Table 2: Decompose by composition: Breakdown function $\mathcal{A}_{\tilde{x}}^{k}(\cdot)_{g}$ for $\pi$ processes (cf. Definition 3.6).

mappings $(\cdot\rangle^{1}, \mathcal{G}(\cdot)$, and $(\cdot\rangle^{2}$, is defined in Fig. 9 (top, where omitted cases are defined homomorphically). It is extended to account for recursive session types in Fig. 9 (center).

The auxiliary function $\mathcal{R}^{\prime \star}(\cdot)$, given in Fig. 9 (bottom), is used in Tab. 2 to decompose guarded tail-recursive types: it skips session prefixes until a type of form $\mu \mathrm{t} . S$ is encountered; when that occurs, the recursive type is decomposed using $\mathcal{R}^{\prime}(\cdot)$.
We are finally ready to define the decomposition function $\mathcal{F}(\cdot)$, the analog of Definition 2.3 but for processes in $\pi$ :

Definition 3.6 (Process Decomposition). Let $P$ be a closed $\pi$ process with $\widetilde{u}=\mathrm{fn}(P)$ and $\widetilde{v}=\mathrm{rn}(P)$. Given the breakdown function $\mathcal{A}_{\tilde{x}}^{k}(\cdot)_{g}$ in Table 2, the decomposition $\mathcal{F}(P)$ is defined as:

$$
\mathcal{F}(P)=(v \widetilde{\mathcal{c}})\left(v \widetilde{c}_{r}\right)\left(\prod_{r \in \tilde{\mathcal{V}}} P^{r}\left|\overline{c_{k}} !\langle\rangle .0\right| \mathcal{A}_{\epsilon}^{k}(P \sigma)_{g}\right)
$$




$$
\begin{aligned}
& \mathcal{H}(\langle S\rangle)=\langle\mathcal{H}(S)\rangle \\
& \mathcal{H}\left(!\langle S\rangle ; S^{\prime}\right)= \begin{cases}M & \text { if } S^{\prime}=\text { end } \\
M, \mathcal{H}\left(S^{\prime}\right) & \text { otherwise }\end{cases} \\
& \text { where } M=!\langle\langle ?(?(\langle ?(\mathcal{H}(S)) \text {; end }\rangle) \text {; end }) \text {; end }\rangle\rangle \text {; end } \\
& \mathcal{H}\left(?(S) ; S^{\prime}\right)= \begin{cases}M & \text { if } S^{\prime}=\text { end } \\
M, \mathcal{H}\left(S^{\prime}\right) & \text { otherwise }\end{cases} \\
& \text { where } M=?(\langle ?(?(\langle ?(\mathcal{H}(S)) \text {; end }\rangle) \text {; end }) \text {; end }\rangle) \text {; end } \\
& \mathcal{H}(\text { end })=\text { end } \\
& \mathcal{H}\left(S_{1}, \ldots, S_{n}\right)=\mathcal{H}\left(S_{1}\right), \ldots, \mathcal{H}\left(S_{n}\right) \\
& \mathcal{H}(\mu \mathrm{t} . S)= \begin{cases}\mathcal{R}^{\prime}(S) & \text { if } \mu \mathrm{t} . S \text { is tail-recursive } \\
\mu \mathrm{t} . \mathcal{H}(S) & \text { otherwise }\end{cases} \\
& \mathcal{R}^{\prime}\left(!\langle S\rangle ; S^{\prime}\right)=\mu \mathrm{t} . !\langle\langle ?(?(\langle ?(\mathcal{H}(S)) ; \text { end }\rangle) ; \text { end }) ; \text { end }\rangle\rangle ; \mathrm{t}, \mathcal{R}^{\prime}\left(S^{\prime}\right) \\
& \mathcal{R}^{\prime}\left(?(S) ; S^{\prime}\right)=\mu \mathrm{t} . ?(\langle ?(?(\langle ?(\mathcal{H}(S)) ; \text { end }\rangle) ; \text { end }) ; \text { end }\rangle) ; \mathrm{t}, \mathcal{R}^{\prime}\left(S^{\prime}\right) \\
& \mathcal{H}(\mathrm{t})=\mathrm{t} \quad \mathcal{R}^{\prime}(\mathrm{t})=\epsilon \\
& \mathcal{R}^{\prime \star}\left(?(S) ; S^{\prime}\right)=\mathcal{R}^{\prime \star}\left(S^{\prime}\right) \quad \mathcal{R}^{\prime \star}\left(!\langle S\rangle ; S^{\prime}\right)=\mathcal{R}^{\prime \star}\left(S^{\prime}\right) \\
& \mathcal{R}^{\prime \star}(\mu \mathrm{t} . S)=\mathcal{R}^{\prime \star}(S)
\end{aligned}
$$

Figure 9: Decomposition of types $\mathcal{H}(\cdot)$ (cf. Def. 3.5)

where: $k>0, \widetilde{c}=\left(c_{k}, \ldots, c_{k+\lfloor P\rceil-1}\right) ; \widetilde{c_{r}}=\bigcup_{r \in \tilde{v}} c^{r} ; \sigma=\{\operatorname{init}(\tilde{u}) / \tilde{u}\}$; $P^{r}=c^{r} ?(b) .(v s)(b !\langle s\rangle . \bar{s} !\langle\widetilde{r}\rangle)$ with $r: S$ and $\widetilde{r}=r_{1}, \ldots, r_{|\mathcal{G}(S)|}$.

\subsection{Examples}

Example 3.7 (A Process with Delegation). Consider a process $P$ that implements channels $\bar{w}$ (with type $T=$ ?(Int);!〈Bool $\rangle$; end) and $u$ (with type $S=!\langle\bar{T}\rangle$; end):

$$
P=(v u: S)(\underbrace{u !\langle w\rangle \cdot \bar{w} ?(t) \cdot \bar{w} !\langle\operatorname{odd}(t)\rangle .0}_{A} \mid \underbrace{\bar{u} ?(x) \cdot x !\langle 5\rangle \cdot x ?(b) .0}_{B})
$$

By Def. 3.2, $\lfloor P\rceil=25$. Then, the decomposition of $P$ into a collection of first-order processes typed with minimal session types is:

$$
\mathcal{F}(P)=\left(v c_{1}, \ldots, c_{25}\right)\left(\overline{c_{1}} !\langle\rangle .0 \mid\left(v u_{1}\right) \mathcal{A}_{\epsilon}^{1}\left((A \mid B) \sigma^{\prime}\right)\right),
$$

where $\sigma=\operatorname{init}(\mathrm{fn}(P))$ and $\sigma^{\prime}=\sigma \cdot\left\{u_{1} \overline{u_{1}} / u \bar{u}\right\}$. We omit parameter $g$ as it is empty. We have:

$$
\mathcal{A}_{\epsilon}^{1}\left((A \mid B) \sigma^{\prime}\right)=c_{1} ?() \cdot \overline{c_{2}} !\langle\rangle \cdot \overline{c_{13}} !\langle\rangle\left|\mathcal{A}_{\epsilon}^{2}\left(A \sigma^{\prime}\right)\right| \mathcal{A}_{\epsilon}^{13}\left(B \sigma^{\prime}\right)
$$

We use the following abbreviations for subprocesses of $A$ and $B$ : $A^{\prime}=\bar{w}_{1} ?(t) \cdot A^{\prime \prime}, A^{\prime \prime}=\bar{w}_{1} !\langle\operatorname{odd}(t)\rangle .0$, and $B^{\prime}=x_{1} !\langle 5\rangle \cdot x_{1} ?(b) .0$. The breakdown of $A$ is in Fig. 10; the breakdown of $B$ follows:

$$
\begin{gathered}
\mathcal{A}_{\epsilon}^{13}(B)=c_{13} ?() \cdot \bar{u}_{1} ?\left(y_{4}\right) \cdot \overline{c_{14}} !\left\langle y_{4}\right\rangle \mid\left(v s_{1}\right)\left(c_{14} ?(y) \cdot \overline{c_{15}} !\langle y\rangle \cdot \overline{c_{16}} !\langle\rangle \mid\right. \\
c_{15} ?\left(y_{4}\right) \cdot\left(v s^{\prime \prime}\right)\left(y_{4} !\left\langle s^{\prime \prime}\right\rangle \cdot \overline{s^{\prime \prime}} !\left\langle s_{1}\right\rangle \cdot 0\right) \mid c_{16} ?() \cdot\left(v a_{3}\right)\left(s_{1} !\left\langle a_{3}\right\rangle .\right. \\
\left.\left.\left(\overline{c_{21}} !\langle\rangle\left|c_{21} ?() \cdot 0\right| a_{3} ?\left(y_{5}\right) \cdot y_{5} ?\left(x_{1}, x_{2}\right) \cdot\left(\overline{c_{17}} !\langle\rangle \mid \mathcal{A}_{\epsilon}^{17}\left(B^{\prime}\right)\right)\right)\right)\right)
\end{gathered}
$$

$$
\begin{aligned}
& \mathcal{A}_{\epsilon}^{2}(A)=c_{2} ?() \cdot\left(v a_{1}\right)\left(u _ { 1 } ! \langle a _ { 1 } \rangle \cdot \left(\overline{c_{5}} !\langle\rangle\left|\mathcal{A}_{\epsilon}^{5}\left(A^{\prime}\right)\right|\right.\right. \\
& a_{1} ?\left(y_{1}\right) \cdot y_{1} ?\left(z_{1}\right) \cdot \overline{c_{3}} !\left\langle z_{1}\right\rangle\left|c_{3} ?\left(z_{1}\right) \cdot z_{1} ?(x) \cdot \overline{c_{4}} !\langle x\rangle\right| \\
& \left.\left.c_{4} ?(x) \cdot(v s)\left(x !\langle s\rangle \cdot \bar{s} !\left\langle w_{1}, w_{2}\right\rangle\right)\right)\right) \\
& \mathcal{A}_{\epsilon}^{5}\left(A^{\prime}\right)=c_{5} ?() \cdot \overline{w_{1}} ?\left(y_{2}\right) \cdot \overline{c_{6}} !\left\langle y_{2}\right\rangle \mid\left(v s_{1}\right)\left(c_{6} ?\left(y_{2}\right) \cdot \overline{c_{7}} !\left\langle y_{2}\right\rangle \cdot \overline{c_{8}} !\langle\rangle \mid\right. \\
& \left.\quad c_{7} ?\left(y_{2}\right) \cdot\left(v s^{\prime}\right)\left(y_{2} !\left\langle s^{\prime}\right\rangle \cdot \overline{s^{\prime}} !\left\langle s_{1}\right\rangle \cdot 0\right)\right) \mid \\
& \quad c_{8} ?() \cdot\left(v a_{2}\right)\left(\overline { s _ { 1 } } ! \langle a _ { 2 } \rangle \cdot \left(\overline{c_{10}} !\langle\rangle\left|c_{10} ?() \cdot \mathbf{0}\right|\right.\right. \\
& \left.\left.\quad a_{2} ?\left(y_{3}\right) \cdot y_{3} ?\left(t_{1}\right) \cdot\left(\overline{c_{9}} !\langle\rangle \mid \mathcal{A}_{\epsilon}^{9}\left(A^{\prime \prime}\right)\right)\right)\right) \\
& \mathcal{A}_{\epsilon}^{9}\left(A^{\prime \prime}\right)=c_{9} ?() \cdot(v a)\left(\overline { w } _ { 2 } ! \langle a \rangle \cdot \left(\overline{c_{12}} !\langle\rangle\left|c_{12} ?() \cdot \mathbf{0}\right|\right.\right. \\
& \quad a ?(y) \cdot y ?\left(z_{1}\right) \cdot \overline{c_{11}} !\left\langle z_{1}\right\rangle\left|c_{10} ?\left(z_{1}\right) \cdot z_{1} ?(x) \cdot \overline{c_{11}} !\langle x\rangle\right| \\
& \left.\left.c_{11} ?(x) \cdot(v s)(x !\langle s\rangle \cdot \bar{s} !\langle\operatorname{odd}(t)\rangle)\right)\right)
\end{aligned}
$$

Figure 10: Breakdown for process $A$ in Exam. 3.7.

The breakdown of $B^{\prime}$ is similar. Type $S$ is broken down into MSTs $M_{1}$ and $M_{2}$, as follows:

$$
\begin{aligned}
& M_{1}=?(\langle ?(?(\langle ?(\text { Int }) ; \text { end }\rangle) ; \text { end }) ; \text { end }\rangle) ; \text { end } \\
& M_{2}=!\langle\langle ?(?(\langle ?(\text { Bool }) ; \text { end }\rangle) ; \text { end }) ; \text { end }\rangle\rangle ; \text { end }
\end{aligned}
$$

Names $\bar{w}_{1}$ and $\bar{w}_{2}$ are typed with $M_{1}$ and $M_{2}$, respectively. Then, name $u_{1}$ is typed with $M$, given by:

$$
M=!\left\langle\left\langle ?\left(?\left(\left\langle ?\left(\bar{M}_{1}, \bar{M}_{2}\right) ; \text { end }\right\rangle\right) ; \text { end }\right) ; \text { end }\right\rangle\right\rangle ; \text { end }
$$

Consider the reductions of $\mathcal{F}(P)$ that mimic the exchange of $w$ along $u$ in $P$. We first have three synchronizations on $c_{1}, c_{2}, c_{13}$ :

$$
\begin{aligned}
& \mathcal{F}(P) \longrightarrow{ }^{3}(v \widetilde{c})\left(( v a _ { 1 } ) \left(u _ { 1 } ! \langle a _ { 1 } \rangle \cdot \left(\overline{c_{5}} !\langle\rangle\left|\mathcal{A}_{\epsilon}^{5}\left(A^{\prime}\right)\right|\right.\right.\right. \\
& a_{1} ?\left(y_{1}\right) \cdot y_{1} ?\left(z_{1}\right) \cdot \overline{c_{3}} !\left\langle z_{1}\right\rangle\left|c_{3} ?\left(z_{1}\right) \cdot z_{1} ?(x) \cdot \overline{c_{4}} !\langle x\rangle\right| \\
&\left.\left.c_{4} ?(x) \cdot(v s)\left(x !\langle s\rangle \cdot \bar{s} !\left\langle w_{1}, w_{2}\right\rangle\right)\right)\right)\left|\overline{u_{1}} ?(y 4) \cdot \overline{c_{14}} !\left\langle y_{4}\right\rangle\right| \\
&\left(v s_{1}\right)\left(c_{14} ?(y) \cdot \overline{c_{15}} !\langle y\rangle \cdot \overline{c_{16}} !\langle\rangle \mid\right. \\
& \quad c_{15} ?\left(y_{4}\right) \cdot\left(v s^{\prime \prime}\right)\left(y_{4} !\left\langle s^{\prime \prime}\right\rangle \cdot \overline{s^{\prime \prime}} !\left\langle s_{1}\right\rangle \cdot 0\right) \mid \\
& c_{16} ?() \cdot\left(v a_{3}\right)\left(s _ { 1 } ! \langle a _ { 3 } \rangle \cdot \left(\overline{c_{21}} !\langle\rangle \mid\right.\right. \\
&\left.\left.\left.\quad c_{21} ?() \cdot 0 \mid a_{3} ?\left(y_{5}\right) \cdot y_{5} ?\left(x_{1}, x_{2}\right) \cdot\left(\overline{c_{17}} !\langle\rangle \mid \mathcal{A}_{\epsilon}^{17}\left(B^{\prime}\right)\right)\right)\right)\right)
\end{aligned}
$$

where $\widetilde{c}=\left(c_{3}, \ldots, c_{12}, c_{14}, \ldots, c_{25}\right)$. Then, a synchronization on $u_{1}$ sends name $a_{1}$ (highlighted above). Name $a_{1}$ is further propagated along $c_{14}$ and $c_{15}$. Another synchronization occurs on $c_{16}$.

$$
\begin{gathered}
\mathcal{F}(P) \longrightarrow^{7}\left(v{\widetilde{c_{*}}}\right)\left(v a_{1}\right)\left(\overline{c_{5}} !\langle\rangle\left|\mathcal{A}_{\epsilon}^{5}\left(A^{\prime}\right)\right| a_{1} ?\left(y_{1}\right) \cdot y_{1} ?\left(z_{1}\right) \cdot \overline{c_{3}} !\left\langle z_{1}\right\rangle \mid\right. \\
c_{3} ?\left(z_{1}\right) \cdot z_{1} ?(x) \cdot \overline{c_{4}} !\langle x\rangle\left|c_{4} ?(x) \cdot(v s)\left(x !\langle s\rangle \cdot \bar{s} !\left\langle w_{1}, w_{2}\right\rangle\right)\right| \\
\left(v s_{1}\right)\left(\left(v s^{\prime \prime}\right)\left(a_{1} !\left\langle s^{\prime \prime}\right\rangle \cdot \overline{s^{\prime \prime}} !\left\langle s_{1}\right\rangle \cdot 0\right) \mid\left(v a_{3}\right)\left(s _ { 1 } ! \langle a _ { 3 } \rangle \cdot \left(\overline{c_{21}} !\langle\rangle \mid\right.\right.\right. \\
\left.\left.\left.\left.c_{21} ?() \cdot 0 \mid a_{3} ?\left(y_{5}\right) \cdot y_{5} ?\left(x_{1}, x_{2}\right) \cdot\left(\overline{c_{17}} !\langle\rangle \mid \mathcal{A}_{\epsilon}^{17}\left(B^{\prime}\right)\right)\right)\right)\right)\right) \\
\text { where } \widetilde{c}_{*}=\left(c_{3}, \ldots, c_{12}, c_{17}, \ldots, c_{25}\right)
\end{gathered}
$$


The next reduction communicates session name $s^{\prime \prime}$ along $a_{1}$ :

$$
\begin{aligned}
\mathcal{F}(P) & \longrightarrow^{8}\left(v{\widetilde{c_{*}}}\right)\left(v s^{\prime \prime}\right)\left(\overline{c_{5}} !\langle\rangle\left|\mathcal{A}_{\epsilon}^{5}\left(A^{\prime}\right)\right|\right. \\
& s^{\prime \prime} ?\left(z_{1}\right) \cdot \overline{c_{3}} !\left\langle z_{1}\right\rangle\left|c_{3} ?\left(z_{1}\right) \cdot z_{1} ?(x) \cdot \overline{c_{4}} !\langle x\rangle\right| \\
& c_{4} ?(x) \cdot(v s)\left(x !\langle s\rangle \cdot \bar{s} !\left\langle w_{1}, w_{2}\right\rangle\right) \mid \\
& \left(v s_{1}\right)\left(\overline{s^{\prime \prime}} !\left\langle s_{1}\right\rangle \cdot 0 \mid\left(v a_{3}\right)\left(s _ { 1 } ! \langle a _ { 3 } \rangle \cdot \left(\overline{c_{21}} !\langle\rangle\left|c_{21} ?() \cdot 0\right|\right.\right.\right. \\
& \left.\left.\left.\left.a_{3} ?\left(y_{5}\right) \cdot y_{5} ?\left(x_{1}, x_{2}\right) \cdot\left(\overline{c_{17}} !\langle\rangle \mid \mathcal{A}_{\epsilon}^{17}\left(B^{\prime}\right)\right)\right)\right)\right)\right)
\end{aligned}
$$

After the synchronization on channel $s^{\prime \prime}$, name $z_{1}$ is further sent to the next parallel process through the propagator $c_{3}$ :

$$
\begin{gathered}
\mathcal{F}(P) \longrightarrow^{10}\left(v{\widetilde{c_{*} *}}\right)\left(v s_{1}\right)\left(\overline{c_{5}} !\langle\rangle\left|\mathcal{A}_{\epsilon}^{5}\left(A^{\prime}\right)\right|\right. \\
s_{1} ?(x) . \overline{c_{4}} !\langle x\rangle\left|c_{4} ?(x) \cdot(v s)\left(x !\langle s\rangle . \bar{s} !\left\langle w_{1}, w_{2}\right\rangle\right)\right| \\
\left(v a_{3}\right)\left(s_{1} !\left\langle a_{3}\right\rangle .\left(\overline{c_{21}} !\langle\rangle\left|c_{21} ?() .0\right|\right.\right. \\
\left.\left.\left.a_{3} ?\left(y_{5}\right) \cdot y_{5} ?\left(x_{1}, x_{2}\right) \cdot\left(\overline{c_{17}} !\langle\rangle \mid \mathcal{A}_{\epsilon}^{17}\left(B^{\prime}\right)\right)\right)\right)\right) \\
\text { where } \widetilde{c}_{* *}=\left(c_{4}, \ldots, c_{12}, c_{17}, \ldots, c_{25}\right)
\end{gathered}
$$

Communication on $s_{1}$ leads to variable $x$ being substituted by name $a_{3}$, which is then passed on $c_{4}$ to the next process. In addition, inaction is simulated by a synchronization on $c_{21}$.

$$
\begin{aligned}
\mathcal{F}(P) \longrightarrow & { }^{13} \\
& \left(v \widetilde{c}_{\bullet}\right)\left(v a_{3}\right)\left(\overline{c_{5}} !\langle\rangle\left|\mathcal{A}_{\epsilon}^{5}\left(A^{\prime}\right)\right|(v s)\left(a_{3} !\langle s\rangle . \bar{s} !\left\langle w_{1}, w_{2}\right\rangle\right) \mid\right. \\
& \left.a_{3} ?\left(y_{5}\right) \cdot y_{5} ?\left(x_{1}, x_{2}\right) \cdot\left(\overline{c_{17}} !\langle\rangle \mid \mathcal{A}_{\epsilon}^{17}\left(B^{\prime}\right)\right)\right) \\
& \text { where } \widetilde{c}_{\bullet}=\left(c_{5}, \ldots, c_{12}, c_{17}, \ldots, c_{25}\right)
\end{aligned}
$$

Now, the passing of the decomposition of $w$ is finally simulated by two reductions: first, a synchronization on $a_{3}$ sends the endpoint of session $s$, which replaces variable $y_{5}$; then, the dual endpoint is used to send $w_{1}, w_{2}$, substituting variables $x_{1}, x_{2}$ in $\mathcal{A}_{\epsilon}^{17}\left(B^{\prime}\right)$.

$$
\begin{aligned}
& \mathcal{F}(P) \longrightarrow{ }^{14}\left(v \widetilde{c}_{\bullet \bullet}\right)(v s)\left(\overline{c_{5}} !\langle\rangle\left|\mathcal{A}_{\epsilon}^{5}\left(A^{\prime}\right)\right|\right. \\
& \left.\bar{s} !\left\langle w_{1}, w_{2}\right\rangle \mid s ?\left(x_{1}, x_{2}\right) .\left(\overline{c_{17}} !\langle\rangle \mid \mathcal{A}_{\epsilon}^{17}\left(B^{\prime}\right)\right)\right) \\
& \mathcal{F}(P) \longrightarrow{ }^{15}\left(v \widetilde{c}_{\bullet \bullet}\right)\left(\overline{c_{5}} !\langle\rangle\left|\mathcal{A}_{\epsilon}^{5}\left(A^{\prime}\right)\right|\right. \\
& \left.\overline{c_{17}} !\langle\rangle \mid \mathcal{A}_{\epsilon}^{17}\left(B^{\prime}\right)\left\{w_{1} w_{2} / x_{1} x_{2}\right\}\right)=Q
\end{aligned}
$$

Above, $\widetilde{c}_{\bullet \bullet}=\left(c_{5}, \ldots, c_{12}, c_{17}, \ldots, c_{25}\right)$. This is how $\mathcal{F}(P)$ simulates the first action of $P$. Notice that in $Q$ names $w_{1}, w_{2}$ substitute $x_{1}, x_{2}$ and the first synchronization on $w$ can be simulated on name $w_{1}$.

Example 3.8 (A Recursive Process). Let $P=\mu X . P^{\prime}$ be a process implementing a channel $r$ with the tail-recursive session type $S=$ $\mu$ t.?(Int); ! Int $\rangle$; t, with $P^{\prime}=r ?(w) . r !\langle-w\rangle . X$. We decompose $r$ using $S$ and obtain two channels typed with MSTs as in Fig. 9:

$$
\begin{aligned}
& r_{1}: \mu \mathrm{t} . ?(\langle ?(?(\langle ?(\text { Int }) ; \text { end }\rangle) ; \text { end }) ; \text { end }\rangle) ; \mathrm{t} \\
& r_{2}: \mu \mathrm{t} . !\langle\langle ?(?(\langle ?(\text { Int }) ; \text { end }\rangle) ; \text { end }) ; \text { end }\rangle) ; \mathrm{t}
\end{aligned}
$$

Then, process $\mathcal{F}(P)$ is

$$
(v \widetilde{c})\left(v c^{r}\right)\left(c^{r} ?(b) .(v s)\left(b !\langle s\rangle . \bar{s} !\left\langle r_{1}, r_{2}\right\rangle\right)\left|\overline{c_{1}} !\langle\rangle\right| \mathcal{A}_{\epsilon}^{1}\left(P\left\{r_{1} / r\right\}\right)_{\emptyset}\right)
$$

where $\widetilde{c}=\left(c_{1}, \ldots, c_{\lfloor P\rceil}\right)$ and $\mathcal{A}_{\epsilon}^{1}\left(P\left\{r_{1} / r\right\}\right)_{\emptyset}$ is in Fig. 11 .

In Fig. 11, $\mathcal{A}_{\epsilon}^{1}\left(P\left\{r_{1} / r\right\}\right)$ simulates recursion in $P$ using replication. Given some index $k$, process $R^{k}$ mimics actions of the recursive body. It first gets a decomposition of $r$ by interacting with the

$$
\begin{aligned}
& \mathcal{A}_{\epsilon}^{1}\left(P\left\{r_{1} / r\right\}\right)_{\emptyset}=\left(v s_{1}\right)\left(c_{1} ?() \cdot \overline{c_{2}} !\langle\rangle \cdot \overline{c_{4}} !\langle\rangle \mid\right. \\
& c_{2} ?() \cdot\left(v a_{1}\right)\left(s _ { 1 } ! \langle a _ { 1 } \rangle \cdot \left(\overline{c_{3}} !\langle\rangle\left|c_{3} ?() \cdot 0\right|\right.\right. \\
& c_{4} ?() \cdot s_{1} ?\left(z_{x}\right) \cdot \overline{c_{5}} !\left\langle z_{x}\right\rangle \mid \\
&\left.\left.\left.R^{5} \mid * a_{1} ?\left(y_{1}^{\prime}\right) \cdot y_{1}^{\prime} ?\left(x_{r_{1}}, x_{r_{2}}, y_{1}\right) \cdot \widehat{P}\right)\right)\right)
\end{aligned}
$$

where:

$$
\begin{aligned}
& \widehat{P}=(v \widetilde{c})\left(c^{r} ?(b) \cdot\left(v s^{\prime}\right)\left(b !\left\langle s^{\prime}\right\rangle \cdot \bar{s}^{\prime} !\left\langle x_{r_{1}}, x_{r_{2}}\right\rangle\right)\left|\overline{c_{1}} !\langle\rangle\right|\right. \\
& \left.c_{1} ?() \cdot y_{1} ?\left(z_{x}\right) \cdot \overline{c_{2}} !\left\langle z_{x}\right\rangle \mid R^{2}\left\{x_{r_{1}}, x_{r_{2}} / r_{1}, r_{2}\right\}\right) \\
& R^{k}=c_{k} ?\left(z_{x}\right) . \\
& \left(v a_{1}\right)\left(c ^ { r } ! \langle a _ { 1 } \rangle \cdot \left(( v s _ { 1 } ) \left(c_{k+1} ?(y) \cdot \overline{c_{k+2}} !\langle y\rangle \cdot \overline{c_{k+3}} !\langle\rangle \mid\right.\right.\right. \\
& c_{k+2} ?(y) \cdot(v s)\left(y !\langle s\rangle . \bar{s} !\left\langle s_{1}\right\rangle\right) \mid \\
& c_{k+3} ?() \cdot\left(v a_{2}\right)\left(s _ { 1 } ! \langle a _ { 2 } \rangle \cdot \left(\overline{c_{k+l+4}} !\langle\rangle \mid\right.\right. \\
& c_{k+l+4} ?() .0 \mid a_{2} ?\left(y_{2}\right) \cdot y_{2} ?\left(w_{1}\right) \text {. } \\
& \left.\left.(v \widetilde{c})\left(\overline{c_{k+4}} !\left\langle z_{x}\right\rangle \mid \mathcal{A}_{z_{x}}^{k+4}\left(r_{2} !\left\langle-w_{1}\right\rangle . X\right)_{g}\right)\right)\right) \mid \\
& a_{1} ?\left(y_{1}\right) \cdot y_{1} ?\left(z_{1}, z_{2}\right) \cdot z_{1} ?(y) \text {. } \\
& \left.\left.\left.\overline{c_{k+1}} !\langle y\rangle \cdot c^{r} ?(b) .\left(v s^{\prime}\right)\left(b !\left\langle s^{\prime}\right\rangle \cdot \bar{s}^{\prime} !\left\langle z_{1}, z_{2}\right\rangle\right)\right)\right)\right) \\
& \mathcal{A}_{z_{x}}^{k+4}\left(r_{2} !\left\langle-w_{1}\right\rangle \cdot X\right)_{g}=c_{k} ?\left(z_{x}\right) \cdot\left(v a_{1}\right) c^{r} !\left\langle a_{1}\right\rangle \text {. } \\
& \left(\mathcal{A}_{z_{x}}^{k+7}(X)_{g} \mid a_{1} ?\left(y_{1}\right) \cdot y_{1} ?(\widetilde{z}) \cdot W\right) \\
& W=\left(\begin{array}{ll}
v & a_{2}
\end{array}\right)\left(z_{2} !\left\langle a_{2}\right\rangle .\left(\overline{c_{k+7}} !\left\langle z_{x}\right\rangle \cdot c^{r} ?(b) .(v s)(b !\langle s\rangle . \bar{s} !\langle\bar{z}\rangle) \mid\right.\right. \\
& a_{2} ?\left(y_{2}\right) \cdot y_{2} ?\left(z_{1}^{\prime}\right) \cdot(v \widetilde{c})\left(\overline{c_{k+5}} !\langle\rangle \mid\right. \\
& c_{k+5} ?() \cdot z_{1}^{\prime} ?(x) \cdot \overline{c_{k+6}} !\langle x\rangle \mid \\
& \left.\left.\left.c_{k+6} ?(x) \cdot\left(v s^{\prime}\right)\left(x !\left\langle s^{\prime}\right\rangle . \bar{s}^{\prime} !\langle-w 1\rangle\right)\right)\right)\right) \\
& \mathcal{A}_{z_{x}}^{k+7}(X)_{g}=\left(v s_{1}\right)\left(c_{k+7} ?\left(z_{x}\right) \cdot \overline{c_{k+8}} !\left\langle z_{x}\right\rangle \cdot \overline{c_{k+9}} !\left\langle z_{x}\right\rangle \mid\right. \\
& c_{k+8} ?\left(z_{x}\right) \cdot\left(v a_{1}\right)\left(c ^ { r } ! \langle a _ { 1 } \rangle \cdot \left(c_{k+9} ?\left(z_{x}\right) \cdot \bar{s}_{1} !\left\langle z_{x}\right\rangle \cdot \mid\right.\right. \\
& \overline{c_{k+10}} !\langle\rangle c_{k+10} ?() .0 \mid \\
& a_{1} ?\left(y_{1}\right) \cdot y_{1} ?\left(r_{1}, r_{2}\right) \text {. } \\
& \left.\left.\left.\left.\left.\left(v s^{\prime}\right)\left(z_{x} !\left\langle s^{\prime}\right\rangle \cdot \bar{s}^{\prime} !\left\langle r_{1}, r_{2}, s_{1}\right\rangle\right)\right)\right)\right)\right)\right)
\end{aligned}
$$

with $g=\left\{X \mapsto r_{1}, r_{2}\right\}$

Figure 11: Breakdown of recursive process (Exam. 3.8)

process providing recursive names on $c^{r}$ (for the first instance, this is a top-level process in $\mathcal{F}(P)$ ). Then, it mimics the first input action on the channel received for $z_{1}$ (that is, $r_{1}$ ): the input of actual names for $w_{1}$ is delegated through channel redirections to name $y_{2}$ (both prefixes are highlighted in Fig. 11). Once the recursive name is used, the decomposition of recursive name is made available for the breakdown of the continuation by a communication on $c^{r}$. Similarly, in the continuation, the second action on $r$, output, is mimicked by $r_{2}$ (received for $z_{2}$ ), with the output of actual name $w_{1}$ delegated to $\bar{s}^{\prime}$ (both prefixes are highlighted in Fig. 11).

Subprocess $R^{5}$ is a breakdown of the first instance of the recursive body. The replication guarded by $a_{1}$ produces a next instance, i.e., process $R^{2}\left\{x_{r_{1}}, x_{r_{2}} / r_{1}, r_{2}\right\}$ in $\widehat{P}$. By communication on $a_{1}$ and a few reductions on propagators, it gets activated: along $a_{1}$ it first receives a name for $y_{1}^{\prime}$ along which it also receives: (i) recursive names 
$r_{1}, r_{2}$ for variables $x_{r_{1}}, x_{r_{2}}$, and (ii) a name for $y_{1}$ along which it will receive $a_{1}$ again, for future instances, as it can be seen in $\mathcal{A}_{z_{x}}^{k+7}(X)_{g}$.

\subsection{Results}

We establish the minimality result for $\pi$ using the typability of $\mathcal{F}(\cdot)$. We need some auxiliary definitions to characterize the propagators required to decompose recursive processes.

Theorems 3.10 and 3.27 in [2] state typability results by introducing two typing environments, denoted $\Theta$ and $\Phi$. While environment $\Theta$ is used to type linear propagators (e.g., $c_{k}, c_{k+1}, \ldots$ ) generated by the breakdown function $\mathcal{B}_{-}^{-}(\cdot)$, environment $\Phi$ types shared propagators used in trios that propagate breakdown of recursive names (e.g., $c^{r}, c^{v}, \ldots$ where $r$ and $v$ are recursive names).

Definition 3.9 (Session environment for propagators). Let $\Theta$ be the session environment and $\Phi$ be the recursive propagator environment defined in Theorem 3.10 and Theorem 3.27 [2], respectively. Then, by applying the encoding $(\cdot\rangle^{2}$, we define $\Theta^{\prime}$ and $\Phi^{\prime}$ as follows: $\Theta^{\prime}=\langle\Theta\rangle^{2}, \Phi^{\prime}=\langle\Phi\rangle^{2}$.

We can use $\Theta^{\prime}=\langle\Theta\rangle^{2}$ in the following statement, where we state the typability result for the breakdown function.

Theorem 3.10 (Typability of Breakdown). Let $P$ be an initialized $\pi$ process. If $\Gamma ; \Delta, \Delta_{\mu} \vdash P \triangleright \diamond$, then

$$
\mathcal{H}\left(\Gamma^{\prime}\right), \Phi^{\prime} ; \mathcal{H}(\Delta), \Theta^{\prime}+\mathcal{A}_{\epsilon}^{k}(P)_{g} \triangleright \diamond
$$

where $k>0 ; \tilde{r}=\operatorname{dom}\left(\Delta_{\mu}\right) ; \Phi^{\prime}=\prod_{r \in \tilde{r}} c^{r}:\left\langle\left\langle ?\left(\mathcal{R}^{\prime \star}\left(\Delta_{\mu}(r)\right)\right)\right.\right.$; end $\left.\rangle\right\rangle$; and balanced $\left(\Theta^{\prime}\right)$ with

$$
\operatorname{dom}\left(\Theta^{\prime}\right)=\left\{c_{k}, c_{k+1}, \ldots, c_{k+\lfloor P\rceil-1}\right\} \cup\left\{\overline{c_{k+1}}, \ldots, \overline{c_{k+\lfloor P\rceil-1}}\right\}
$$

such that $\Theta^{\prime}\left(c_{k}\right)=?(\cdot)$; end.

Proof. Directly by using Theorem 5.1 [11], Theorem 3.27 [2], and Theorem 5.2 [11]. See [1] for details.

We now consider typability for the decomposition function, using $\Phi^{\prime}=\langle\Phi\rangle^{2}$ as in Def. 3.9. The proof follows from Thm. 3.10; see [1].

Theorem 3.11 (Minimality Result for $\pi$ ). Let $P$ be a closed $\pi$ process, with $\widetilde{u}=\mathrm{fn}(P)$ and $\widetilde{v}=\mathrm{rn}(P)$. If $\Gamma ; \Delta, \Delta_{\mu} \vdash P \triangleright \diamond$, where $\Delta_{\mu}$ only involves recursive session types, then $\mathcal{H}(\Gamma \sigma) ; \mathcal{H}(\Delta \sigma), \mathcal{H}\left(\Delta_{\mu} \sigma\right) \vdash \mathcal{F}(P) \triangleright \diamond$, where $\sigma=\{\operatorname{init}(\widetilde{u}) / \widetilde{u}\}$.

\section{OPTIMIZATIONS}

Although conceptually simple, the composition approach to decomposition induces redundancies. Here we propose $\mathcal{F}^{*}(\cdot)$, an optimization of the decomposition $\mathcal{F}(\cdot)$, and establish its static and dynamic correctness, in terms of the minimality result (cf. Thm. 4.14) but also operational correspondence (cf. Thm. 4.20), respectively.

\subsection{Motivation}

To motivate our insights, consider the process $\mathcal{A}_{\tilde{x}}^{k}\left(u_{i} ?(w) . Q\right)_{g}$ as presented in $\S 3.1$ and Tab. 2. We identify some suboptimal features of this decomposition: (i) channel redirections; (ii) redundant synchronizations on propagators; (iii) the structure of trios is lost.

While an original process $P$ receives a name for variable $w$ along $u_{i}$, its breakdown does not input a breakdown of $w$ directly, but through a series of channel redirections: $u_{i}$ receives a name along which it sends restricted name $s$, along which it sends the restricted name $s_{1}$ and so on. Finally, the name received for $y^{\prime}$ receives $\widetilde{w}$, the breakdown of $w$. This redundancy is perhaps more evident in Def. 3.5, which gives the translation of types by composition: the mimicked input action is five-level nested for the original name. This is due to the composition of $\llbracket \cdot \rrbracket_{g}^{1}$ and $\llbracket \cdot \rrbracket^{2}$.

Also, $\mathcal{A}_{\tilde{x}}^{k}\left(u_{i} ?(w) \cdot Q\right)_{g}$ features redundant communications on propagators. For example, the bound name $y$ is locally propagated by $c_{k+1}$ and $c_{k+2}$. This is the result of breaking down sequential prefixes induced by $\llbracket \cdot \rrbracket_{g}^{1}$ (not present in the original process). Last but not least, the trio structure is lost as subprocess $\widehat{Q}_{\tilde{x}}$ is guarded and nested, and it inductively invokes the function on continuation $Q$. This results in an arbitrary level of process nesting, which is induced by the final application of encoding $\llbracket \cdot \rrbracket^{2}$ in the composition.

The non-optimality of $\mathcal{A}_{\tilde{x}}^{k}(\cdot)_{g}$ is more prominent in the treatment of recursive processes and recursive names. As $\mathrm{HO}$ does not feature recursion constructs, $\llbracket \cdot \rrbracket_{g}^{1}$ encodes recursive behaviors by relying on abstraction passing and shared abstractions. Then, going back to $\pi$ via $\llbracket \cdot \rrbracket_{g}^{2}$, this is translated to a process involving a replicated subprocess. But going through this path, the encoding of recursive process becomes convoluted. On top of that, all nonoptimal features of the core fragment (as discussed for the case of input) are also present in the decomposition of recursion.

Here we develop an optimized decomposition function, denoted $\mathcal{F}^{*}(\cdot)$ (Def. 4.8), that avoids the redundancies described above. The optimized decomposition produces a composition of trios processes, with a fixed maximum number of nested prefixes. The decomposed process does not redirect channels and only introduces propagators that codify the sequentiality of the original process.

\subsection{Preliminaries}

We decompose a session type into a list of minimal session types:

Definition 4.1 (Decomposing Types). Let $S$ and $C$ be a session and a channel type, resp. (cf. Fig. 4). The type decomposition function $\mathcal{H}^{*}(\cdot)$ is defined in Figure 12.

Example 4.2 (Decomposing a Recursive Type). Let $S=\mu \mathrm{t} . S^{\prime}$ be a recursive session type, with $S^{\prime}=$ ?(Int); ?(Bool); ! 〈Bool $\rangle$; t. By Fig. 12, since $S$ is tail-recursive, $\mathcal{H}^{*}(S)=\mathcal{R}\left(S^{\prime}\right)$. Further,

$$
\mathcal{R}\left(S^{\prime}\right)=\mu \mathrm{t} . ?\left(\mathcal{H}^{*}(\operatorname{lnt})\right) ; \mathrm{t}, \mathcal{R}(?(\mathrm{Bool}) ; !\langle\text { Bool }\rangle ; \mathrm{t})
$$

By definition of $\mathcal{R}(\cdot)$, we obtain

$$
\mathcal{H}^{*}(S)=\mu \mathrm{t} \text {.?(Int); t, } \mu \mathrm{t} \text {.?(Bool); t, } \mu \mathrm{t} \text {.! }\langle\text { Bool }\rangle ; \mathrm{t}, \mathcal{R}(t)
$$

(using $\mathcal{H}^{*}($ Int $)=$ Int and $\mathcal{H}^{*}(\mathrm{Bool})=$ Bool). Since $\mathcal{R}(\mathrm{t})=\epsilon$, we have

$$
\mathcal{H}^{*}(S)=\mu \mathrm{t} . ?(\text { Int }) ; \mathrm{t}, \mu \mathrm{t} . ?(\mathrm{Bool}) ; \mathrm{t}, \mu \mathrm{t} . !\langle\mathrm{Bool}\rangle ; \mathrm{t}
$$

Example 4.3 (Decomposing an Unfolded Recursive Type). Let $T=$ ?(Bool); ! 〈Bool $\rangle ; S$ be a derived unfolding of $S$ from Exam. 4.2. Then, by Fig. $12, \mathcal{R}^{\star}(T)$ is the list of minimal recursive types obtained as follows: first, $\mathcal{R}^{\star}(T)=\mathcal{R}^{\star}\left(\right.$ ! $\langle$ Bool $\left.\rangle ; \mu \mathrm{t} . S^{\prime}\right)$ and after one more step, $\mathcal{R}^{\star}\left(!\langle\right.$ Bool $\left.\rangle ; \mu \mathrm{t} . S^{\prime}\right)=\mathcal{R}^{\star}\left(\mu \mathrm{t} . S^{\prime}\right)$. Finally, we have $\mathcal{R}^{\star}\left(\mu \mathrm{t} . S^{\prime}\right)=$ $\mathcal{R}\left(S^{\prime}\right)$. We get the same list of minimal types as in Exam. 4.2:

$$
\mathcal{R}^{\star}(T)=\mu \mathrm{t} \text {.?(Int); t, } \mu \mathrm{t} \text {.?(Bool); }, \mu \mathrm{t} . \text { ! }\langle\text { Bool }\rangle ; \mathrm{t}
$$




$$
\begin{aligned}
\mathcal{H}^{*}(\text { end }) & =\text { end } \\
\mathcal{H}^{*}(\langle S\rangle) & =\left\langle\mathcal{H}^{*}(S)\right\rangle \\
\mathcal{H}^{*}\left(S_{1}, \ldots, S_{n}\right) & =\mathcal{H}^{*}\left(S_{1}\right), \ldots, \mathcal{H}^{*}\left(S_{n}\right) \\
\mathcal{H}^{*}(!\langle C\rangle ; S) & = \begin{cases}!\left\langle\mathcal{H}^{*}(C)\right\rangle ; \text { end } & \text { if } S=\text { end } \\
!\left\langle\mathcal{H}^{*}(C)\right\rangle ; \text { end }, \mathcal{H}^{*}(S) & \text { otherwise }\end{cases} \\
\mathcal{H}^{*}(?(C) ; S) & = \begin{cases}?\left(\mathcal{H}^{*}(C)\right) ; \text { end } & \text { if } S=\text { end } \\
?\left(\mathcal{H}^{*}(C)\right) ; \text { end }, \mathcal{H}^{*}(S) & \text { otherwise }\end{cases} \\
\mathcal{H}^{*}\left(\mu \mathrm{t} . S^{\prime}\right) & =\mathcal{R}\left(S^{\prime}\right) \\
\mathcal{H}^{*}(S) & =\mathcal{R}^{\star}(S) \quad \text { where } S \neq \mu \mathrm{t} . S^{\prime} \\
\mathcal{R}(\mathrm{t}) & =\epsilon \\
\mathcal{R}(!\langle C\rangle ; S) & =\mu \mathrm{t} . !\left\langle\mathcal{H}^{*}(C)\right\rangle ; \mathrm{t}, \mathcal{R}(S) \\
\mathcal{R}(?(C) ; S) & =\mu \mathrm{t} . ?\left(\mathcal{H}^{*}(C)\right) ; \mathrm{t}, \mathcal{R}(S) \\
\mathcal{R}^{\star}(?(C) ; S) & =\mathcal{R}^{\star}(!\langle C\rangle ; S)=\mathcal{R}{ }^{\star}(S) \\
\mathcal{R}^{\star}(\mu \mathrm{t} . S) & =\mathcal{R}(S)
\end{aligned}
$$

\section{Figure 12: Decomposition of types $\mathcal{H}^{*}(\cdot)$ (cf. Def. 4.1)}

Definition 4.4 (Decomposing Environments). Given environments $\Gamma$ and $\Delta$, we define $\mathcal{H}^{*}(\Gamma)$ and $\mathcal{H}^{*}(\Delta)$ inductively as $\mathcal{H}^{*}(\emptyset)=\emptyset$ and

$$
\begin{aligned}
\mathcal{H}^{*}\left(\Delta, u_{i}: S\right) & =\mathcal{H}^{*}(\Delta),\left(u_{i}, \ldots, u_{i+\left|\mathcal{H}^{*}(S)\right|-1}\right): \mathcal{H}^{*}(S) \\
\mathcal{H}^{*}\left(\Gamma, u_{i}:\langle S\rangle\right) & =\mathcal{H}^{*}(\Gamma), u_{i}: \mathcal{H}^{*}(\langle S\rangle)
\end{aligned}
$$

Definition 4.5 (Degree of a Process). The optimized degree of a process $P$, denoted $\lfloor P\rceil^{*}$, is inductively defined as follows:

$$
\begin{cases}\lfloor Q\rceil^{*}+1 & \text { if } P=u_{i} !\langle y\rangle \cdot Q \text { or } P=u_{i} ?(y) \cdot Q \\ \lfloor Q\rceil^{*} & \text { if } P=(v s: S) Q \\ \lfloor Q\rceil^{*}+1 & \text { if } P=(v r: S) Q \text { and } \operatorname{tr}(S) \\ \lfloor Q\rceil^{*}+\lfloor R\rceil^{*}+1 & \text { if } P=Q \mid R \\ 1 & \text { if } P=0 \text { or } P=X \\ \lfloor Q\rceil^{*}+1 & \text { if } P=\mu X \cdot Q\end{cases}
$$

As before, given a finite tuple of names $\widetilde{u}=\left(a, b, s, s^{\prime}, \ldots\right)$, we write init $(\widetilde{u})$ to denote the tuple $\left(a_{1}, b_{1}, s_{1}, s_{1}^{\prime}, \ldots\right)$; also, we say that a process is initialized if all of its names have some index.

Given a tuple of initialized names $\tilde{u}$ and a tuple of indexed names $\tilde{x}$, it is useful to collect those names in $\widetilde{x}$ that appear in $\widetilde{u}$.

Definition 4.6 (Free indexed names). Let $\widetilde{u}$ and $\widetilde{x}$ be two tuples of names. We define the set $\operatorname{fnb}(\widetilde{u}, \widetilde{x})$ as $\left\{z_{k}: z_{i} \in \widetilde{u} \wedge z_{k} \in \widetilde{x}\right\}$.

As usual, we treat sets of names as tuples (and vice-versa). By abusing notation, given a process $P$, we shall write $\operatorname{fnb}(P, \widetilde{y})$ to stand for $\mathrm{fnb}(\mathrm{fn}(P), \widetilde{y})$. Then, we have that $\mathrm{fnb}(P, \widetilde{x}) \subseteq \widetilde{x}$. In the definition of the breakdown function, this notion allows us to conveniently determine a context for a subsequent trio.

REMARK 1. Whenever $c_{k}$ ? $(\widetilde{y})\left(\right.$ resp. $\left.\overline{c_{k}} !\langle\widetilde{y}\rangle\right)$ with $\widetilde{y}=\epsilon$, we shall write $c_{k}$ ?() (resp. $\left.\overline{c_{k}} !\langle\rangle\right)$ to stand for $c_{k}$ ? $(y)$ (resp. $\left.\overline{c_{k}} !\langle y\rangle\right)$ such that $c_{k}: ?(\langle$ end $\rangle)$; end (resp. $\overline{c_{k}}: !\langle\langle$ end $\rangle\rangle$; end).
Definition 4.7 (Index function). Let $S$ be an (unfolded) recursive session type. The function $f(S)$ is defined as follows:

$$
f(S)= \begin{cases}f_{0}^{\prime}\left(S^{\prime}\{S / \mathrm{t}\}\right) & \text { if } S=\mu \mathrm{t} . S^{\prime} \\ f_{0}^{\prime}(S) & \text { otherwise }\end{cases}
$$

where: $f_{l}^{\prime}(!\langle U\rangle ; S)=f_{l+1}^{\prime}(S), f_{l}^{\prime}(?(U) ; S)=f_{l+1}^{\prime}(S)$, and $f_{l}^{\prime}(\mu \mathrm{t} . S)=|\mathcal{R}(S)|-l+1$.

Given a process $P$, we write $\operatorname{frv}(P)$ to denote that $P$ has a free recursive variable.

\subsection{The Optimized Decomposition}

We define the optimized decomposition $\mathcal{F}^{*}(\cdot)$ by relying on the revised breakdown function $\mathbb{A}_{\tilde{x}}^{k}(\cdot)$ (cf. $\left.\S 4.3 .1\right)$. Given a context $\tilde{x}$ and a $k>0, \mathbb{A}_{\tilde{x}}^{k}(\cdot)$ is defined on initialized processes. Table 3 gives the definition: we use an auxiliary function for recursive processes, denoted $\mathbb{A}_{\text {rec } \tilde{x}}^{k}(\cdot)_{g}($ cf. $\S 4.3 .2)$, where parameter $g$ is a mapping from recursive variables to a list of name variables.

In the following, to keep presentation simple, we assume processes $\mu X . P$ in which $P$ does not contain a subprocess of shape $\mu Y . P^{\prime}$. The generalization of our decomposition without this assumption is not difficult, but is notationally heavy.

4.3.1 The Breakdown Function. We describe entries 1-7 in Table 3. 1. Input Process $\mathbb{A}_{\tilde{x}}^{k}\left(u_{i} ?(y) . Q\right)$ consists of a leading trio that mimics the input and runs in parallel with the breakdown of $Q$. In the trio, a context $\tilde{x}$ is expected along $c_{k}$. Then, an input on $u_{l}$ mimics the input action: it expects the decomposition of name $y$, denoted $\widetilde{y}$. To decompose $y$ we use its type: if $y: S$ then $\widetilde{y}=\left(y_{1}, \ldots, y_{\left|\mathcal{H}^{*}(S)\right|}\right)$. The index of $u_{l}$ depends on the type of $u_{i}$. Intuitively, if $u_{i}$ is tailrecursive then $l=f(S)$ (Def. 4.7) as index and we do not increment it, as the same decomposition of $u_{i}$ should be used to mimic a new instance in the continuation. Otherwise, if $u_{i}$ is linear then we use the substitution $\sigma=\left\{u_{i+1} / u_{i}\right\}$ to increment it in $Q$. Next, the context $\widetilde{z}=\mathrm{fnb}(Q, \widetilde{x y} \backslash \widetilde{w})$ is propagated, where $\widetilde{w}=\left(u_{i}\right)$ or $\widetilde{w}=\epsilon$. 2. Output Process $\mathbb{A}_{\tilde{x}}^{k}\left(u_{i} !\left\langle y_{j}\right\rangle \cdot Q\right)$ sends the decomposition of $y$ on $u_{l}$, with $l$ as in the input case. We decompose name $y_{j}$ based on its type $S: \widetilde{y}=\left(y_{j}, \ldots, y_{j+\left|\mathcal{H}^{*}(S)\right|-1}\right)$. The context to be propagated is $\widetilde{z}=\mathrm{fnb}(P, \widetilde{x} \backslash \widetilde{w})$, where $\widetilde{w}$ and $\sigma$ are as in the input case.

3. Restriction (Non-recursive name) The breakdown of process $(v s: C) Q$ is $\left(v \widetilde{s}: \mathcal{H}^{*}(C)\right) \mathcal{B}_{\tilde{x}}^{k}(Q \sigma)$, where $s$ is decomposed using $C: \widetilde{s}=\left(s_{1}, \ldots, s_{\left|\mathcal{H}^{*}(C)\right|}\right)$. Since $(v s)$ binds $s$ and its dual $\bar{s}$ (or only $s$ if $C$ is a shared type) the substitution $\sigma$ is simply $\left\{s_{1} \bar{s}_{1} / s \bar{s}\right\}$ and initializes indexes in $Q$.

4. Restriction (Recursive name) As in the previous case, in the breakdown of $(v s: \mu$ t.S)Q the name $s$ is decomposed into $\widetilde{s}$ by relying on $\mu \mathrm{t}$.S. Here the breakdown consists of the breakdown of $Q$ running in parallel with a control trio, which appends restricted (recursive) names $\widetilde{s}$ and $\widetilde{\bar{s}}$ to the context, i.e., $\widetilde{z}=\widetilde{x}, \widetilde{s}, \widetilde{\bar{s}}$.

5. Composition The breakdown of process $Q_{1} \mid Q_{2}$ uses a control trio to trigger the breakdowns of $Q_{1}$ and $Q_{2}$, similarly as before.

6. Inaction The breakdown of 0 is simply an input prefix that receives an empty context (i.e., $\widetilde{x}=\epsilon$ ).

7. Recursion The breakdown of $\mu X . P$ is as follows:

$$
\left(v c_{X}^{r}\right)\left(c_{k} ?(\widetilde{x}) \cdot \overline{c_{k+1}^{r}} !\langle\widetilde{z}\rangle \cdot \mu X \cdot c_{X}^{r} ?(\widetilde{y}) \cdot \overline{c_{k+1}^{r}} !\langle\widetilde{y}\rangle \cdot X \mid \mathbb{A}_{\text {rec } \left.\tilde{z}^{k+1}(P)_{g}\right)}\right)
$$




\begin{tabular}{|c|c|c|c|}
\hline & $P$ & $\mathbb{A}_{\tilde{x}}^{k}(P)$ & \\
\hline 1 & $u_{i} ?(y) \cdot Q$ & $c_{k} ?(\widetilde{x}) \cdot u_{l} ?(\widetilde{y}) \cdot \overline{c_{k+1}} !\langle\widetilde{z}\rangle \mid \mathbb{A}_{\tilde{z}}^{k+1}(Q \sigma)$ & $\begin{array}{l}y_{j}: S \wedge \widetilde{y}=\left(y_{1}, \ldots, y_{|S|}\right) \\
\widetilde{w}=\left(\operatorname{lin}\left(u_{i}\right)\right) ?\left\{u_{i}\right\}: \epsilon \\
\widetilde{z}=\operatorname{fnb}(Q, \widetilde{x} \backslash \backslash \widetilde{w}) \\
l=\left(\operatorname{tr}\left(u_{i}\right)\right) ? f(S): i \\
\sigma=\operatorname{next}\left(u_{i}\right) \cdot\left\{y_{1} / y\right\}\end{array}$ \\
\hline 2 & $u_{i} !\left\langle y_{j}\right\rangle \cdot Q$ & $c_{k} ?(\widetilde{x}) \cdot u_{l} !\langle\widetilde{y}\rangle \cdot \overline{c_{k+1}} !\langle\widetilde{z}\rangle \mid \mathbb{A}_{\tilde{z}}^{k+1}(Q \sigma)$ & $\begin{array}{l}y_{j}: S \wedge \widetilde{y}=\left(y_{j}, \ldots, y_{j+\left|\mathcal{H}^{*}(S)\right|-1}\right) \\
\widetilde{w}=\left(\operatorname{lin}\left(u_{i}\right)\right) ?\left\{u_{i}\right\}: \epsilon \\
\widetilde{z}=\operatorname{fnb}(Q, \widetilde{x} \backslash \widetilde{w}) \\
l=\left(\operatorname{tr}\left(u_{i}\right)\right) ? f(S): i \\
\sigma=\operatorname{next}\left(u_{i}\right)\end{array}$ \\
\hline 3 & $(v s: C) Q$ & $\left(v \widetilde{s}: \mathcal{H}^{*}(C)\right) \mathbb{A}_{\tilde{x}}^{k}(Q \sigma)$ & $\begin{array}{l}\widetilde{s}=\left(s_{1}, \ldots, s_{\left|\mathcal{H}^{*}(C)\right|}\right) \\
\sigma=\left\{s_{1} \overline{s_{1}} / s \bar{s}\right\}\end{array}$ \\
\hline 4 & $(v s: \mu \mathrm{t} . S) Q$ & $(v \widetilde{s}: \mathcal{R}(S))\left(c_{k} ?(\widetilde{x}) \cdot \overline{c_{k+1}} !\langle\widetilde{z}\rangle \cdot 0 \mid \mathbb{A}_{\tilde{z}}^{k+1}(Q)\right)$ & 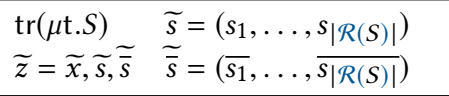 \\
\hline 5 & $Q_{1} \mid Q_{2}$ & $c_{k} ?(\widetilde{x}) \cdot \overline{c_{k+1}} !\langle\widetilde{y}\rangle \cdot \overline{c_{k+l+1}} !\langle\widetilde{z}\rangle\left|\mathbb{A}_{\tilde{y}}^{k+1}\left(Q_{1}\right)\right| \mathbb{A}_{\tilde{z}}^{k+l+1}\left(Q_{2}\right)$ & $\begin{array}{l}\widetilde{y}=f \mathrm{nb}\left(Q_{1}, \widetilde{x}\right) \quad \widetilde{z}=\mathrm{fnb}\left(Q_{2}, \widetilde{x}\right) \\
l=\left|Q_{1}\right|\end{array}$ \\
\hline 6 & 0 & $c_{k} ?() .0$ & \\
\hline 7 & $\mu X . P$ & $\left(v c_{X}^{r}\right)\left(c_{k} ?(\widetilde{x}) \cdot \overline{c_{k+1}^{r}} !\langle\widetilde{z}\rangle \cdot \mu X \cdot c_{X}^{r} ?(\widetilde{y}) \cdot \overline{c_{k+1}^{r}} !\langle\widetilde{y}\rangle \cdot X \mid \mathbb{A}_{r e c}^{k+1} \tilde{z}^{(P)_{g}}\right)$ & $\begin{array}{ll}\widetilde{n}=\mathrm{fs}(P) & \widetilde{n}: \widetilde{C} \wedge \widetilde{z}=\operatorname{bn}(\widetilde{n}: \widetilde{C}) \\
|\widetilde{z}|=|\widetilde{y}| & g=\{X \mapsto \widetilde{z}\}\end{array}$ \\
\hline & $P$ & $\mathbb{A}_{\text {rec } \tilde{x}}^{k}(P)_{g}$ & \\
\hline 8 & $u_{i} !\left\langle y_{j}\right\rangle \cdot Q$ & $\begin{array}{ll}\mu X \cdot c_{k}^{r} ?(\widetilde{x}) \cdot u_{l} !\langle\widetilde{y}\rangle \cdot \bar{c}_{k+1}^{r} !\langle\widetilde{z}\rangle \cdot X \mid \mathbb{A}_{\text {rec } \tilde{z}^{k+1}(Q \sigma)_{g}} & (\text { if } g \neq \emptyset) \\
\mu X \cdot c_{k}^{r} ?(\widetilde{x}) \cdot\left(u_{l} !\langle\widetilde{y}\rangle \cdot c_{k+1}^{r} !\langle\widetilde{z}\rangle \mid X\right) \mid \mathbb{A}_{\text {rec } \tilde{z}}^{k+1}(Q \sigma)_{g} & (\text { if } g=\emptyset)\end{array}$ & $\begin{array}{l}y: T \wedge\left(y_{j}, \ldots, y_{j+\left|\mathcal{H}^{*}(T)\right|-1}\right) \\
\widetilde{w}=\left(\operatorname{lin}\left(u_{i}\right)\right) ?\left\{u_{i}\right\}: \epsilon \\
\widetilde{z}=g(X) \cup \operatorname{fnb}(Q, \widetilde{x} \backslash \widetilde{w}) \\
l=\left(\operatorname{tr}\left(u_{i}\right)\right) ? f(S): i \\
\sigma=\operatorname{next}\left(u_{i}\right)\end{array}$ \\
\hline 9 & $u_{i} ?(y) \cdot Q$ & $\begin{array}{ll}\mu X \cdot c_{k}^{r} ?(\widetilde{x}) \cdot u_{l} ?(\widetilde{y}) \cdot \overline{c_{k+1}^{r}} !\langle\widetilde{z}\rangle \cdot X \mid \mathbb{A}_{\operatorname{rec} \tilde{z}}^{k+1}(Q \sigma)_{g} & (\text { if } g \neq \emptyset) \\
\mu X . c_{k}^{r} ?(\widetilde{x}) \cdot\left(u_{l} ?(\widetilde{y}) \cdot c_{k+1}^{r} !\langle\widetilde{z}\rangle \mid X\right) \mid \mathbb{A}_{\operatorname{rec} \tilde{z}}^{k+1}(Q \sigma)_{g} & (\text { if } g=\emptyset)\end{array}$ & $\begin{array}{l}\widetilde{w}=\left(\operatorname{lin}\left(u_{i}\right)\right) ?\left\{u_{i}\right\}: \epsilon \\
\widetilde{z}=g(X) \cup \operatorname{fnb}(Q, \widetilde{x y} \backslash \widetilde{w}) \\
l=\left(\operatorname{tr}\left(u_{i}\right)\right) ? f(S): i \\
\sigma=\operatorname{next}\left(u_{i}\right) \cdot\left\{y_{1} / y\right\}\end{array}$ \\
\hline 10 & $Q_{1} \mid Q_{2}$ & 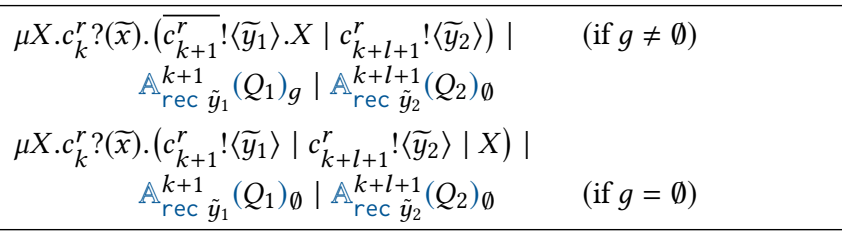 & $\begin{array}{l}\operatorname{frv}\left(Q_{1}\right) \\
\widetilde{y}_{1}=g(X) \cup \mathrm{fnb}\left(Q_{1}, \widetilde{x}\right) \\
\widetilde{y}_{2}=\operatorname{fnb}\left(Q_{2}, \widetilde{x}\right) \\
l=\left|Q_{1}\right|\end{array}$ \\
\hline 11 & $(v s: C) Q$ & $\begin{array}{ll}\mu X .\left(v \widetilde{s}: \mathcal{H}^{*}(C)\right) c_{k}^{r} ?(\widetilde{x}) \cdot \bar{c}_{k+1}^{r} !\langle\widetilde{z}\rangle \cdot X \mid \mathbb{A}_{r \text { ec } \tilde{z}}^{k+1}(Q \sigma)_{g} & (\text { if } g \neq \emptyset) \\
\mu X .\left(v \widetilde{s}: \mathcal{H}^{*}(C)\right) c_{k}^{r} ?(\widetilde{x}) \cdot\left(c_{k+1}^{r} !\langle\widetilde{z}\rangle \mid X\right) \mid \mathbb{A}_{r \text { ec }}^{k+1}(Q \sigma)_{g} & (\text { if } g=\emptyset)\end{array}$ & $\begin{array}{l}\widetilde{s}=\left(s_{1}, \ldots, s_{\left|\mathcal{H}^{*}(S)\right|}\right) \\
\widetilde{\bar{s}}=(\operatorname{lin}(S)) ?\left(\overline{s_{1}}, \ldots, \overline{s_{\mid \mathcal{H}}(S) \mid}\right): \epsilon \\
\widetilde{z}=\widetilde{x}, \widetilde{s}, \overline{\bar{s}} \quad \sigma=\left\{s_{1} \overline{s_{1}} / s \bar{s}\right\}\end{array}$ \\
\hline 12 & $X$ & $\begin{array}{ll}\mu X \cdot c_{k}^{r} ?(\widetilde{x}) \cdot c_{X}^{r} !\langle\widetilde{x}\rangle X & (\text { if } g \neq \emptyset) \\
\mu X . c_{k}^{r} ?() \cdot\left(c_{X}^{r} !\langle\rangle \mid X\right) & (\text { if } g=\emptyset)\end{array}$ & \\
\hline 13 & 0 & $c_{k}^{r} ?() .0$ & \\
\hline
\end{tabular}

Table 3: Optimized breakdown function $\mathbb{A}_{\tilde{x}}^{k}(\cdot)$ for processes, and auxiliary function for recursive processes $\mathbb{A}_{\text {rec }}^{k}(\cdot)_{g}$.

We have a control trio and the breakdown of $P$, obtained using $\mathbb{A}_{\text {rec } \tilde{x}}^{k}(\cdot)_{g}(\S 4.3 .2)$. The trio receives the context $\widetilde{x}$ on $c_{k}$ and propagates it further. To ensure typability, we bind all session free names of $P$ using the context $\widetilde{z}$, which contains the decomposition of those free names. This context is needed to break down $P$, and so we record it as $g=\{X \mapsto \widetilde{z}\}$ in the definition of $\mathbb{A}_{\text {rec } \tilde{x}}^{k+1}(P)_{g}$. This way, $\widetilde{z}$ will be propagated all the way until reaching $X$.

Next, the recursive trio is enabled, and receives $\widetilde{y}$ along $c_{X}^{r}$, with $|\widetilde{z}|=|\widetilde{y}|$ and $l=|P|$. The tuple $\widetilde{y}$ is propagated to the first trio of $\mathbb{A}_{\text {rec } \tilde{x}}^{k+1}(P)_{g}$. By definition of $\mathbb{A}_{\text {rec } \tilde{x}}^{k+1}(P)_{g}$, its propagator $c_{X}^{r}$ will 
send the same context as received by the first trio. Hence, the recursive part of the control trio keeps sending this context to the next instances of recursive trios of $\mathbb{A}_{r e c}^{k+1}(P)_{g}$.

Notice that the leading trio actually has four prefixes. This simplifies our presentation: this trio can be broken down into two trios by introducing an extra propagator $c_{k+1}$ to send over $c_{k+2}^{r}$.

4.3.2 Handling $P$ in $\mu X . P$. As already mentioned, we use the auxiliary function $\mathbb{A}_{\text {rec } \tilde{x}}^{k}(\cdot)_{g}$ to generate recursive trios.

We discuss entries 8-11 in Tab. 3 (other entries are similar as before). A key observation is that parameter $g$ can be empty. To see this, consider a process like $P=\mu X .\left(Q_{1} \mid Q_{2}\right)$ where $X$ occurs free in $Q_{1}$ but not in $Q_{2}$. If $X$ occurs free in $Q_{1}$ then its decomposition will have a non-empty $g$, whereas the $g$ for $Q_{2}$ will be empty. In the recursive trios of Tab. 3 , the difference between $g \neq \emptyset$ and $g=\emptyset$ is subtle: in the former case, $X$ appears guarded by a propagator; in the latter case, it appears unguarded in a parallel composition. This way, trios in the breakdown of $Q_{2}$ replicate themselves on a trigger from the breakdown of $Q_{1}$.

Given this difference, we only describe the cases when $g \neq \emptyset$ :

8 / 9. Output and Input The breakdown of $u_{i} !\left\langle y_{j}\right\rangle \cdot Q$ consists of the breakdown of $Q$ in parallel with a leading trio, a recursive process whose body is defined as in $\mathcal{B}(\cdot)$. As names $g(X)$ may not appear free in $Q$, we must ensure that a context $\widetilde{z}$ for the recursive body is propagated. The breakdown of $r$ ? $(y) . Q$ is defined similarly.

10. Parallel Composition We discuss the breakdown of $Q_{1} \mid Q_{2}$ assuming $\operatorname{frv}\left(Q_{1}\right)$. We take $\widetilde{y}_{1}=g(X) \cup \operatorname{fnb}\left(Q_{1}, \widetilde{x}\right)$ to ensure that $g(X)$ is propagated to the breakdown of $X$. The role of $c_{k+l+1}^{r}$ is to enact a new instance of the breakdown of $Q_{2}$; it has a shared type to enable replication. In a running process, the number of these triggers in parallel denotes the number of available instances of $Q_{2}$

11. Recursive Variable In this case, the breakdown is a control trio that receives the context $\widetilde{x}$ from a preceding trio and propagates it again to the first control trio of the breakdown of a recursive process along $c_{X}^{r}$. Notice that by construction we have $\widetilde{x}=g(X)$.

We may now define the optimized process decomposition:

Definition 4.8 (Decomposing Processes, Optimized). Let $P$ be a $\pi$ process with $\widetilde{u}=\mathrm{fn}(P)$ and $\widetilde{v}=\mathrm{rn}(P)$. Given the breakdown function $\mathbb{A}_{\tilde{x}}^{k}(\cdot)$ in Table 3 , the optimized decomposition of $P$, denoted $\mathcal{F}^{*}(P)$, is defined as

$$
\mathcal{F}^{*}(P)=(v \widetilde{c})\left(\overline{c_{k}} !\langle\widetilde{r}\rangle .0 \mid \mathbb{A}_{\tilde{r}}^{k}(P \sigma)\right)
$$

where: $k>0 ; \widetilde{c}=\left(c_{k}, \ldots, c_{k+\lfloor P\rceil^{*}-1}\right) ; \widetilde{r}$ such that for $v \in \widetilde{v}$ and $v: S\left(v_{1}, \ldots, v_{|\mathcal{R}(S)|}\right) \subseteq \widetilde{r}$; and $\sigma=\{\operatorname{init}(\widetilde{u}) / \widetilde{u}\}$.

\subsection{Examples}

We now illustrate $\mathcal{F}^{*}(\cdot), \mathbb{A}_{\tilde{x}}^{k}(\cdot), \mathbb{A}_{\text {rec } \tilde{x}}^{k}(\cdot)_{g}$, and $\mathcal{H}^{*}(\cdot)$.

Example 4.9 (Exam. 3.7, Revisited). Consider again the process $P=(v u)(A \mid B)$ as in Exam. 3.7. Recall that $P$ implements session types $S=!\langle\bar{T}\rangle$; end and $T=$ ?(Int);!〈 Bool $\rangle$; end.

By Def. 4.5, $\lfloor P\rceil^{*}=9$. The optimized decomposition of $P$ is:

$$
\mathcal{F}^{*}(P)=(v \widetilde{c})\left(\overline{c_{1}} !\langle\rangle \mid\left(v u_{1}\right) \mathbb{A}_{\epsilon}^{1}\left((A \mid B) \sigma^{\prime}\right)\right)
$$

where $\sigma^{\prime}=\operatorname{init}(\mathrm{fn}(P)) \cdot\left\{u_{1} \overline{u_{1}} / u \bar{u}\right\}$ and $\widetilde{c}=\left(c_{1}, \ldots, c_{9}\right)$. We have:

$$
\left.\mathbb{A}_{\epsilon}^{1}\left((A \mid B) \sigma^{\prime}\right)\right)=c_{1} ?() \cdot \overline{c_{2}} !\langle\rangle \cdot \overline{c_{6}} !\langle\rangle\left|\mathbb{A}_{\epsilon}^{2}\left(A \sigma^{\prime}\right)\right| \mathbb{A}_{\epsilon}^{6}\left(B \sigma^{\prime}\right)
$$

The breakdowns of sub-processes $A$ and $B$ are as follows:

$$
\begin{aligned}
\mathbb{A}_{\epsilon}^{2}\left(A \sigma^{\prime}\right)= & c_{2} ?() \cdot u_{1} !\left\langle w_{1}, w_{2}\right\rangle \cdot \overline{c_{3}} !\langle\rangle\left|c_{3} ?() \cdot \bar{w}_{1} ?(t) \cdot \overline{c_{4}} !\langle\rangle\right| \\
& c_{4} ?() \cdot \bar{w}_{2} !\langle\operatorname{odd}(t)\rangle \cdot \overline{c_{5}} !\langle\rangle \mid c_{5} ?() \cdot 0 \\
\mathbb{A}_{\epsilon}^{6}\left(B \sigma^{\prime}\right)= & c_{6} ?() \cdot \bar{u}_{1} ?\left(x_{1}, x_{2}\right) \cdot \overline{c_{7}} !\left\langle x_{1}, x_{2}\right\rangle\left|c_{7} ?\left(x_{1}, x_{2}\right) \cdot x_{1} !\langle 5\rangle \cdot \overline{c_{8}} !\left\langle x_{2}\right\rangle\right| \\
& c_{8} ?\left(x_{2}\right) \cdot x_{2} ?\left(b_{1}\right) \cdot \overline{c_{9}} !\langle\rangle \mid c_{9} ?() \cdot 0
\end{aligned}
$$

Name $w$ is decomposed as indexed names $\bar{w}_{1}, \bar{w}_{2}$; by using $\mathcal{H}^{*}(\cdot)$ (Def. 4.1) on $T$, their MSTs are $M_{1}=$ ! $\langle$ Int $\rangle$; end and $M_{2}=$ ?(Bool); end, respectively. Name $u_{1}$ is the decomposition of name $u$ and it is typed with $!\left\langle\bar{M}_{1}, \bar{M}_{2}\right\rangle$; end. After a few administrative reductions on $c_{1}$, $c_{2}$, and $c_{6}, \mathcal{F}^{*}(P)$ mimics the first source communication:

$$
\begin{gathered}
\mathcal{F}^{*}(P) \longrightarrow{ }^{3}\left(v{\widetilde{c_{*}}}\right)\left(u_{1} !\left\langle w_{1}, w_{2}\right\rangle \cdot \overline{c_{3}} !\langle\rangle\left|\mathbb{A}_{\epsilon}^{3}(\bar{w} ?(t) \cdot \bar{w} !\langle\operatorname{odd}(t)\rangle \cdot \mathbf{0})\right|\right. \\
\left.\bar{u}_{1} ?\left(x_{1}, x_{2}\right) \cdot \overline{c_{7}} !\left\langle x_{1}, x_{2}\right\rangle \mid \mathbb{A}_{x_{1}, x_{2}}^{7}(x !\langle 5\rangle \cdot x ?(b) .0)\right) \\
\longrightarrow\left(v \widetilde{c_{*}}\right)\left(\overline{c_{3}} !\langle\rangle\left|\mathbb{A}_{\epsilon}^{2}(\bar{w} ?(t) \cdot \bar{w} !\langle\operatorname{odd}(t)\rangle \cdot 0)\right| \overline{c_{7}} !\left\langle w_{1}, w_{2}\right\rangle \mid\right. \\
\left.\mathbb{A}_{x_{1}, x_{2}}^{7}(x !\langle 5\rangle \cdot x ?(b) .0)\right)
\end{gathered}
$$

Above, $\widetilde{c}_{*}=\left(c_{3}, c_{4}, c_{5}, c_{7}, c_{8}, c_{9}\right)$. After reductions on $c_{3}$ and $c_{7}$, name $w_{1}$ substitutes $x_{1}$ and the communication along $w_{1}$ can be mimicked:

$$
\begin{gathered}
\mathcal{F}^{*}(P) \longrightarrow^{6}\left(v{\widetilde{c_{* *}}}\right) \bar{w}_{1} ?(t) \cdot \overline{c_{4}} !\langle\rangle\left|c_{4} ?() \cdot \bar{w}_{2} !\langle\operatorname{odd}(t)\rangle \cdot \overline{c_{5}} !\langle\rangle\right| \\
c_{5} ?() \cdot 0\left|w_{1} !\langle 5\rangle \cdot \overline{c_{8}} !\left\langle w_{2}\right\rangle\right| \\
c_{8} ?\left(x_{2}\right) \cdot x_{2} ?\left(b_{1}\right) \cdot \overline{c_{9}} !\langle\rangle \mid c_{9} ?() \cdot 0 \\
\longrightarrow\left(v{\widetilde{c_{* *}}}\right)\left(\overline{c_{4}} !\langle 5\rangle\left|c_{4} ?(t) \cdot \bar{w}_{2} !\langle\operatorname{odd}(t)\rangle \cdot \overline{c_{5}} !\langle\rangle\right| c_{5} ?() \cdot 0 \mid\right. \\
\left.\overline{c_{8}} !\left\langle w_{2}\right\rangle\left|c_{8} ?\left(x_{2}\right) \cdot x_{2} ?\left(b_{1}\right) \cdot \overline{c_{9}} !\langle\rangle\right| c_{9} ?() \cdot \mathbf{0}\right)
\end{gathered}
$$

Above, $\widetilde{c}_{* *}=\left(c_{4}, c_{5}, c_{8}, c_{9}\right)$. Further reductions follow similarly.

Example 4.10 (Example 3.8, Revisited). Consider again the tailrecursive session type $S=\mu \mathrm{t}$.?(Int);!〈Int $\rangle$; t. Also, let $R$ be a process implementing a channel $r$ with type with $S$ as follows:

$$
R=\mu X \cdot R^{\prime} \quad R^{\prime}=r ?(z) \cdot r !\langle-z\rangle \cdot X
$$

We decompose name $r$ using $S$ and obtain two channels typed with MSTs as in Fig. 12. We have: $r_{1}: \mu \mathrm{t}$.?(Int); t and $r_{2}: \mu \mathrm{t}$.! 〈Int $\rangle$; t.

The trios produced by $\mathbb{A}_{\epsilon}^{k}(R)$ satisfy two properties: they (1) mimic the recursive behavior of $R$ and (2) use the same decomposition of channel $r$ (i.e., $r_{1}, r_{2}$ ) in every instance.

To accomplish (1), each trio of the breakdown of the recursion body is a recursive trio. For (2), we need two things. First, we expect to receive all recursive names in the context $\widetilde{x}$ when entering the decomposition of the recursion body; further, each trio should use one recursive name from the names received and propagate all of them to subsequent trio. Second, we need an extra control trio when breaking down prefix $\mu X$ : this trio (i) receives recursive names from the last trio in the breakdown of the recursion body and (ii) activates another instance with these recursive names.

Using these ideas, we have the decomposed process $\mathbb{A}_{r_{1}, r_{2}}^{1}(R)$ :

$$
c_{1} ?\left(r_{1}, r_{2}\right) \cdot \overline{c_{2}^{r}} !\left\langle r_{1}, r_{2}\right\rangle \cdot \mu X \cdot c_{X}^{r} ?\left(y_{1}, y_{2}\right) \cdot \overline{c_{2}^{r}} !\left\langle y_{1}, y_{2}\right\rangle \cdot X \mid \mathbb{A}_{\text {rec } r_{1}, r_{2}}^{2}\left(R^{\prime}\right)
$$


where $\mathbb{A}_{\text {rec } r_{1}, r_{2}}^{2}\left(R^{\prime}\right)$ is the composition of three recursive trios:

$$
\begin{aligned}
& \mu X . c_{2}^{r} ?\left(y_{1}, y_{2}\right) \cdot r_{1} ?\left(z_{1}\right) \cdot \overline{c_{3}^{r}} !\left\langle y_{1}, y_{2}, z_{1}\right\rangle \cdot X \mid \\
& \mu X . c_{3}^{r} ?\left(y_{1}, y_{2}, z_{1}\right) \cdot r_{2} ?\left(-z_{1}\right) \cdot \overline{c_{4}^{r}} !\left\langle y_{1}, y_{2}\right\rangle \cdot X \mid \\
& \mu X . c_{4}^{r} ?\left(y_{1}, y_{2}\right) \cdot \overline{c_{X}^{r}} !\left\langle y_{1}, y_{2}\right\rangle \cdot X
\end{aligned}
$$

$c_{2}^{r}$ will first activate the recursive trios with context $\left(r_{1}, r_{2}\right)$. Next, each trio uses one of $r_{1}, r_{2}$ and propagate them both mimicking the recursion body. The last recursive trio sends $r_{1}, r_{2}$ to the top-level control trio, so it can enact another instance of the decomposition of the recursion body by activating the first recursive trio.

\subsection{Measuring the Optimization}

Here we measure the improvements of $\mathcal{F}^{*}(\cdot)$ over $\mathcal{F}(\cdot)$. A key metric for comparison is the number of prefixes/sychronizations induced by each decomposition. This includes (1) the number of prefixes involved in channel redirections and (2) the number of propagators; both can be counted by already defined notions:

(1) Channel redirections can be counted by the levels of nesting in the decompositions of types (cf. Fig. 9 and Fig. 12)

(2) The number of propagators is determined by the degree of a process (cf. Def. 3.2 and Def. 4.5)

These two metrics are related; let us discuss them in detail.

Channel redirections. The decompositions of types for $\mathcal{F}(\cdot)$ and $\mathcal{F}^{*}(\cdot)$ abstractly describe the respective channel redirections. The type decomposition for $\mathcal{F}(\cdot)$ (Fig. 9) defines 5 levels of nesting for the translation of input/output types. Then, at the level of (decomposed) processes, channels with these types implement redirections: the nesting levels correspond to 5 additional prefixes in the decomposed process that mimic a source input/output action. In contrast, the type decomposition for $\mathcal{F}^{*}(\cdot)$ (Fig. 12) induces no nesting, and so at the level of processes there are no additional prefixes.

Number of propagators. We define auxiliary functions to count the number of propagators induced by $\mathcal{F}(\cdot)$ and $\mathcal{F}^{*}(\cdot)$. These functions, denoted $\#(\cdot)$ and $\#^{*}(\cdot)$, respectively, are defined using the degree functions $(L \cdot\rceil$ and $\left.L \cdot 7^{*}\right)$ given by Def. 3.2 and Def. 4.5.

Remarkably, $L \cdot\rceil$ and $\#(\cdot)$ are not equal. The difference lies in the number of tail-recursive names in a process. In $\mathcal{F}(\cdot)$ there are propagators $c_{k}$ but also $c^{r}$, used for recursive names. Def. 3.2, however, only counts propagators of form $c_{k}$. For any $P$, the number of propagators $c^{r}$ in $\mathcal{F}(P)$ is the number of free and bound tailrecursive names in $P$. We remark that, by definition, there may be more than one occurrence of a propagator $c^{r}$ in $\mathcal{F}(P)$ : there is at least one prefix with subject $c^{r}$; further occurrences depend on the sequencing structure of the (recursive) type assigned to $r$. On the other hand, in $\mathcal{F}^{*}(P)$ there are propagators $c_{k}$ and propagators $c_{X}^{r}$, whose number corresponds to the number of recursive variables in the process. To define $\#(\cdot)$ and $\#^{*}(\cdot)$, we write $\operatorname{brn}(P)$ to denote bound occurrences of recursive names and $\#_{X}(P)$ to denote the number of occurrences of recursive variables.

Definition 4.11 (Propagators in $\mathcal{F}(P)$ and $\mathcal{F}^{*}(P)$ ). Given a process $P$, the number of propagators in each decomposition is given by

$$
\#(P)=\lfloor P\rceil+2 \cdot|\operatorname{brn}(P)|+|\operatorname{rn}(P)| \quad \#^{*}(P)=\lfloor P\rceil^{*}+\#_{X}(P)
$$

Notice that $\#^{*}(P)$ gives the exact number of actions induced by propagators in $\mathcal{F}^{*}(P)$; in contrast, due to propagators $c^{r}$, \#(P) gives the least number of such actions in $\mathcal{F}(P)$.

In general, we have $\#(P) \geq \#^{*}(P)$, but we can be more precise for a broad class of processes. We say that a $\pi$ process $P \not \equiv 0$ is in normal form if $P=(v \widetilde{n})\left(Q_{1}|\ldots| Q_{n}\right)$, where each $Q_{i}$ (with $i \in\{1, \ldots, n\})$ is not $\mathbf{0}$ and does not contain restriction nor parallel composition at top-level. We have the following result; see [1] for details.

Proposition 1. If $P$ is in normal form then $\#(P) \geq \frac{5}{3} \cdot \#^{*}(P)$.

This result implies that the number of (extra) synchronizations induced by propagators in $\mathcal{F}(P)$ is larger than in $\mathcal{F}^{*}(P)$.

\subsection{Results}

4.6.1 Static Correctness. We first state Thm. 4.13, which ensures the typability of $\mathbb{A}_{\tilde{x}}^{k}(\cdot)$ under MSTs. We rely on an auxiliary predicate:

Definition 4.12 (Indexed Names). Suppose some typing environments $\Gamma, \Delta$. Let $\widetilde{x}, \widetilde{y}$ be two tuples of indexed names. We write indexed $_{\Gamma, \Delta}(\widetilde{y}, \widetilde{x})$ for the predicate

$$
\left.\forall z_{i} \cdot\left(z_{i} \in \tilde{x} \Leftrightarrow\left(\left(z_{i}, \ldots, z_{i+m-1}\right) \subseteq \widetilde{y}\right) \wedge m=\left|\mathcal{H}^{*}\left((\Gamma, \Delta)\left(z_{i}\right)\right)\right|\right)\right)
$$

Theorem 4.13 (Typability of Breakdown). Let $P$ be an initialized process. If $\Gamma ; \Delta \vdash P \triangleright \diamond$ then

$$
\mathcal{H}^{*}(\Gamma \backslash \widetilde{x}) ; \mathcal{H}^{*}(\Delta \backslash \widetilde{x}), \Theta \vdash \mathbb{A}_{\tilde{y}}^{k}(P) \triangleright \diamond \quad(k>0)
$$

where $\widetilde{x} \subseteq \mathrm{fn}(P)$ and $\widetilde{y}$ such that indexed $\Gamma, \Delta(\widetilde{y}, \widetilde{x})$. Also, balanced $(\Theta)$ with

$$
\begin{aligned}
& \quad \operatorname{dom}(\Theta)=\left\{c_{k}, c_{k+1}, \ldots, c_{k+|P|-1}\right\} \cup\left\{\overline{c_{k+1}}, \ldots, \overline{c_{k+|P|-1}}\right\} \\
& \text { and } \Theta\left(c_{k}\right)=?(\widetilde{M}) \text {; end, where } \widetilde{M}=\left(\mathcal{H}^{*}(\Gamma), \mathcal{H}^{*}(\Delta)\right)(\widetilde{y})
\end{aligned}
$$

Proof. By induction of the structure of $P$; see [1] for details.

We now (re)state the minimality result, now based on the decomposition $\mathcal{F}^{*}(\cdot)$. The proof follows from Thm. 4.13; see [1].

Theorem 4.14 (Minimality Result for $\pi$, Optimized). Let $P$ be a $\pi$ process with $\tilde{u}=\mathrm{fn}(P)$. If $\Gamma ; \Delta \vdash P \triangleright \diamond$ then $\mathcal{H}^{*}(\Gamma \sigma) ; \mathcal{H}^{*}(\Delta \sigma) \vdash$ $\mathcal{F}^{*}(P) \triangleright \diamond$, where $\sigma=\{\operatorname{init}(\widetilde{u}) / \widetilde{u}\}$.

4.6.2 Dynamic Correctness. As a complement to the minimality result, we have established that $P$ and $\mathcal{F}^{*}(P)$ are behaviorally equivalent (Thm. 4.20). We overview this result and its required notions.

Thm. 4.20 relies on MST-bisimilarity (cf. Def. $4.19, \approx^{\mathrm{M}}$ ), a variant of the characteristic bisimilarity in [10]. We discuss key differences between the two notions. First, we let an action along name $n$ to be mimicked by an action on a possibly indexed name $n_{i}$, for some $i$.

Definition 4.15 (Indexed name). Given a name $n$, we write $\breve{n}$ to either denote $n$ or any indexed name $n_{i}$, with $i>0$.

Suppose we wish to relate $P$ and $Q$ using $\approx^{\mathrm{M}}$, and that $P$ performs an output action involving name $v$. In our setting, $Q$ should send a tuple of names: the decomposition of $v$. The second difference is that output objects should be related by the relation $\bowtie_{C}$ : 
Definition 4.16 (Relating names). Let $\epsilon$ denote the empty list. We define $\triangle_{C}$ as the relation on names defined as

$$
\overline{\epsilon \bowtie_{C} \epsilon} \quad \frac{\Gamma ; \Delta \vdash n_{i} \triangleright C}{n_{i} \bowtie_{C}\left(n_{i}, \ldots, n_{\left.i+\left|\mathcal{H}^{*}(C)\right|-1\right)}\right.} \quad \frac{\tilde{n} \bowtie_{C} \tilde{m}_{1} \quad n_{i} \bowtie_{C} \tilde{m}_{2}}{\tilde{n}, n_{i} \bowtie_{C} \tilde{m}_{1}, \tilde{m}_{2}}
$$

Characteristic bisimilarity equates typed processes by relying on characteristic processes and trigger processes. These notions, which we recall below, need to be adjusted for them to work with MSTs.

Definition 4.17 (Characteristic trigger process [10]). The characteristic trigger process for type $C$ is

$$
t \Leftarrow_{\mathrm{C}} v: C \stackrel{\text { def }}{=} t ?(x) \cdot(v s)\left(s ?(y) \cdot[C]^{y} \mid \bar{s} !\langle v\rangle .0\right)
$$

where $[C]^{y}$ is the characteristic process for $C$ on name $y[10]$.

Our variant of trigger processes is defined as follows:

Definition 4.18 (Minimal characteristic trigger process). Given a type $C$, the trigger process is

$$
t \Leftarrow_{\mathrm{m}} v_{i}: C \stackrel{\text { def }}{=} t_{1} ?(x) \cdot\left(v s_{1}\right)\left(s_{1} ?(\widetilde{y}) \cdot\langle C\rangle_{i}^{y} \mid \overline{s_{1}} !\langle\tilde{v}\rangle .0\right)
$$

where $v_{i} \bowtie_{\mathrm{C}} \widetilde{v}, y_{i} \bowtie_{\mathrm{C}} \widetilde{y}$, and $\langle C\rangle_{i}^{y}$ is a minimal characteristic process for type $C$ on name $y$ (see [1] for a definition).

We are now ready to define MST-bisimilarity:

Definition 4.19 (MST-Bisimilarity). A typed relation $\mathfrak{R}$ is an MST bisimulation if for all $\Gamma_{1} ; \Delta_{1} \vdash P_{1} R \Gamma_{2} ; \Delta_{2} \vdash Q_{1}$,

(1) Whenever $\Gamma_{1} ; \Delta_{1} \vdash P_{1} \stackrel{\left(v \widetilde{m_{1}}\right) n !\left\langle v: C_{1}\right\rangle}{\longrightarrow} \Delta_{1}^{\prime} ; \Lambda_{1}^{\prime} \vdash P_{2}$ then there

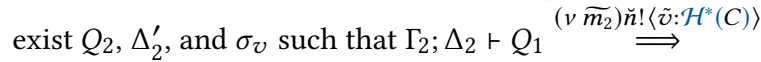
$\Delta_{2}^{\prime} \vdash Q_{2}$ where $v \sigma_{v} \bowtie_{\mathrm{c}} \widetilde{v}$ and, for a fresh $t$,

$$
\begin{gathered}
\Gamma ; \Delta_{1}^{\prime \prime} \vdash\left(v \widetilde{m_{1}}\right)\left(P_{2} \mid t \Leftarrow_{\mathrm{c}} v: C_{1}\right) \Re \\
\Delta_{2}^{\prime \prime} \vdash\left(v \widetilde{m_{2}}\right)\left(Q_{2} \mid t \Leftarrow_{\mathrm{m}} v \sigma: C_{1}\right)
\end{gathered}
$$

(2) Whenever $\Gamma_{1} ; \Delta_{1} \vdash P_{1} \stackrel{n ?(v)}{\longrightarrow} \Delta_{1}^{\prime} \vdash P_{2}$ then there exist $Q_{2}, \Delta_{2}^{\prime}$, and $\sigma_{v}$ such that $\Gamma_{2} ; \Delta_{2} \vdash Q_{1} \stackrel{\check{n} ?(\tilde{v})}{\Longrightarrow} \Delta_{2}^{\prime} \vdash Q_{2}$ where $v \sigma_{v} \bowtie_{\mathrm{C}} \widetilde{v}$ and $\Gamma_{1} ; \Delta_{1}^{\prime} \vdash P_{2} \mathfrak{R} \Gamma_{2} ; \Delta_{2}^{\prime} \vdash Q_{2}$,

(3) Whenever $\Gamma_{1} ; \Delta_{1} \vdash P_{1} \stackrel{\ell}{\longrightarrow} \Delta_{1}^{\prime} \vdash P_{2}$, with $\ell$ not an output or input, then there exist $Q_{2}$ and $\Delta_{2}^{\prime}$ such that $\Gamma_{2} ; \Delta_{2} \vdash Q_{1} \stackrel{\hat{\ell}}{\Longrightarrow}$ $\Delta_{2}^{\prime} \vdash Q_{2}$ and $\Gamma_{1} ; \Delta_{1}^{\prime} \vdash P_{2} \mathfrak{R} \Gamma_{2} ; \Delta_{2}^{\prime} \vdash Q_{2}$ and $\operatorname{sub}(\ell)=n$ implies $\operatorname{sub}(\hat{\ell})=\breve{n}$.

(4) The symmetric cases of 1,2 , and 3.

The largest such bisimulation is called MST bisimilarity $\left(\approx^{\mathrm{M}}\right)$.

We can now state our dynamic correctness result:

Theorem 4.20 (Operational Correspondence). Let $P$ be $a \pi$ process such that $\Gamma_{1} ; \Delta_{1} \vdash P_{1}$. We have

$$
\Gamma ; \Delta \vdash P \approx^{\mathrm{M}} \mathcal{H}^{*}(\Gamma) ; \mathcal{H}^{*}(\Delta) \vdash \mathcal{F}^{*}(P)
$$

Proof. By coinduction: we exhibit a binary relation $\mathcal{S}$ that contains $\left(P, \mathcal{F}^{*}(P)\right)$ and prove that it is an MST bisimulation. See [1] for the full account.

\section{CONCLUDING REMARKS}

Concluding Remarks. We showed a minimality result for $\pi$, a session-typed $\pi$-calculus. This result says that sequentiality in session types is a convenient but not indispensable feature. Following [2], we introduced minimal session types (MSTs) for $\pi$ and defined two decompositions, which transform processes typable with standard session types into processes typable with MSTs. The first decomposition composes existing encodability results and the minimality result for $\mathrm{HO}$; the second decomposition optimizes the first one by (i) removing redundant synchronizations and (ii) using the native support of recursion in $\pi$. For this optimized decomposition, we proved also an operational correspondence result. This way, our work shows that the minimality result is independent from the kind of communicated objects (names or abstractions).

Sequentiality is the key distinguishing feature in the specification of message-passing programs using session types. We remark that by our minimality results do not mean that sequentiality in session types is redundant in modeling and specifying processes; rather, we claim that it is not an indispensable notion to type-checking them. Because we can type-check session typed processes using type systems that do not directly support sequencing in types, our decomposition defines a technique for implementing session types into languages whose type systems do not support sequentiality.

For the sake of space, we have not considered choice constructs (selection and branching). There is no fundamental obstacle in treating them, apart from a very minor caveat: the decomposition in [2] assumes typed processes in which every selection construct comes with a corresponding branching (see [1] for an example).

All in all, besides settling a question left open in [2], our work deepens our understanding about session-based concurrency and the connection between the first-order and higher-order paradigms.

Related Work. We use the trios decomposition by Parrow [12], which he studied for an untyped $\pi$-calculus with replication; in contrast, $\pi$ processes feature recursion. We stress that our goal is to clarify the role of sequentiality in session types by using processes with MSTs, which lack sequentiality. While Parrow's approach elegantly induces processes typable with MSTs, defining trios decompositions for $\pi$ is just one path towards our goal.

Our work differs significantly with respect to [2]. The source language in [2] is $\mathrm{HO}$, based on abstraction-passing, whereas here we focus on the name-passing calculus $\pi$. While in HO propagators carry abstractions, in our case propagators are binding and carry names. Also, names must be decomposed and propagating them requires care. Further novelties appear when decomposing processes with recursion, which require a dedicated collection of recursive trios (not supported in $\mathrm{HO}$ ).

Prior works have related session types with different type systems [3-5]. Loosely related is the work by Dardha et al. [3]. They compile a session $\pi$-calculus down into a $\pi$-calculus with the linear type system of [8] extended with variant types. They represent sequentiality using a continuation-passing style: a session type is interpreted as a linear type carrying a pair consisting of the original payload type and a new linear channel type, to be used for ensuing interactions. The differences are also technical: the approach in [3] thus involves translations connecting two different $\pi$-calculi and 
two different type systems. In contrast, our approach based on MSTs justifies sequentiality using a single typed process framework.

\section{ACKNOWLEDGMENTS}

This work has been partially supported by the Dutch Research Council (NWO) under project No. 016.Vidi.189.046 (Unifying Correctness for Communicating Software).

We are grateful to the anonymous reviewers for their careful reading and constructive remarks.

\section{REFERENCES}

[1] Alen Arslanagic, Anda-Amelia Palamariuc, and Jorge A. Pérez. 2021. Minimal Session Types for the $\pi$-calculus (Extended Version). CoRR abs/2107.10936 (2021) arXiv:2107.10936 http://arxiv.org/abs/2107.10936

[2] Alen Arslanagic, Jorge A. Pérez, and Erik Voogd. 2019. Minimal Session Types (Pearl). In 33rd European Conference on Object-Oriented Programming, ECOOP 2019, fuly 15-19, 2019, London, United Kingdom (LIPIcs, Vol. 134), Alastair F. Donaldson (Ed.). Schloss Dagstuhl - Leibniz-Zentrum für Informatik, 23:1-23:28. https: //doi.org/10.4230/LIPIcs.ECOOP.2019.23

[3] Ornela Dardha, Elena Giachino, and Davide Sangiorgi. 2012. Session types revisited. In Proc. of PPDP 2012. ACM, 139-150. https://doi.org/10.1145/2370776. 2370794

[4] Romain Demangeon and Kohei Honda. 2011. Full Abstraction in a Subtyped piCalculus with Linear Types. In Proc. of CONCUR 2011 (LNCS, Vol. 6901). Springer 280-296. https://doi.org/10.1007/978-3-642-23217-6_19
[5] Simon J. Gay, Nils Gesbert, and António Ravara. 2014. Session Types as Generic Process Types. In Proceedings Combined 21st International Workshop on Expressiveness in Concurrency and 11th Workshop on Structural Operational Semantics, EXPRESS 2014, and 11th Workshop on Structural Operational Semantics, SOS 2014, Rome, Italy, 1st September 2014. (EPTCS, Vol. 160), Johannes Borgström and Silvia Crafa (Eds.). 94-110. https://doi.org/10.4204/EPTCS.160.9

[6] Kohei Honda. 1993. Types for Dyadic Interaction. In CONCUR'93 (LNCS, Vol. 715), Eike Best (Ed.). Springer-Verlag, 509-523.

[7] Kohei Honda, Vasco T. Vasconcelos, and Makoto Kubo. 1998. Language Primitives and Type Disciplines for Structured Communication-based Programming. In ESOP'98 (LNCS, Vol. 1381). Springer, 22-138.

[8] Naoki Kobayashi, Benjamin C. Pierce, and David N. Turner. 1999. Linearity and the Pi-Calculus. TOPLAS 21, 5 (Sept. 1999), 914-947.

[9] Dimitrios Kouzapas, Jorge A. Pérez, and Nobuko Yoshida. 2016. On the Relative Expressiveness of Higher-Order Session Processes. In Programming Languages and Systems - 25th European Symposium on Programming, ESOP 2016, Held as Part of the European foint Conferences on Theory and Practice of Software, ETAPS 2016, Eindhoven, The Netherlands, April 2-8, 2016, Proceedings (Lecture Notes in Computer Science, Vol. 9632), Peter Thiemann (Ed.). Springer, 446-475. https: //doi.org/10.1007/978-3-662-49498-1_18

[10] Dimitrios Kouzapas, Jorge A. Pérez, and Nobuko Yoshida. 2017. Characteristic bisimulation for higher-order session processes. Acta Inf. 54, 3 (2017), 271-341. https://doi.org/10.1007/s00236-016-0289-7

[11] Dimitrios Kouzapas, Jorge A. Pérez, and Nobuko Yoshida. 2019. On the relative expressiveness of higher-order session processes. Inf. Comput. 268 (2019). https: //doi.org/10.1016/j.ic.2019.06.002

[12] Joachim Parrow. 2000. Trios in concert. In Proof, Language, and Interaction, Essays in Honour of Robin Milner, Gordon D. Plotkin, Colin Stirling, and Mads Tofte (Eds.). The MIT Press, 623-638. Online version, dated July 22, 1996, available at http://user.it.uu.se/ joachim/trios.pdf.

[13] Davide Sangiorgi. 1992. Expressing Mobility in Process Algebras: First-Order and Higher Order Paradigms. Ph.D. Dissertation. University of Edinburgh. 